Universidade de São Paulo

\author{
Instituto de Química
}

\title{
Estudo Teórico da Espécie BeMg
}

André Luis Gois Rodrigues

Dissertação de Mestrado

Orientador: Prof. Dr. Antonio Carlos Borin

São Paulo

17 de Abril de 2002 


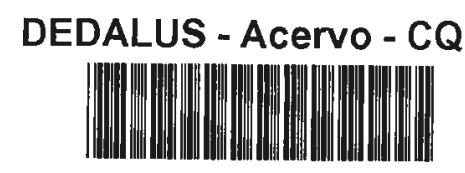

30100004768

Ficha Catalográfica

Elaborada pela Divisão de Biblioteca e

Documentação do Conjunto das Químicas da USP.

\footnotetext{
Rodrigues, André Luis Gois

R696e Estudo teórico da espécie BeMg / André Luis Gois Rodrigues. -- São Paulo, 2002.

$59 \mathrm{p}$.
}

Dissertação (mestrado) - Instituto de Química da Universidade de São Paulo. Departamento de Química Fundamental.

Orientador: Borin, Antonio Carlos

1. Química quântica 2. Estrutura molecular : Química teórica I. T. II. Borin, Antonio Carlos, orientador. 


\section{"Estudo Teórico da Espécie BeMg"}

\section{ANDRÉ LUIS GOIS RODRIGUES}

Dissertação de Mestrado submetida ao Instituto de Ouímica da Universidade de São Paulo como parte dos requisitos necessários à obtenção do grau de Mestre em Ouímica - Área: Físico-Ouímica

Aprovado por:

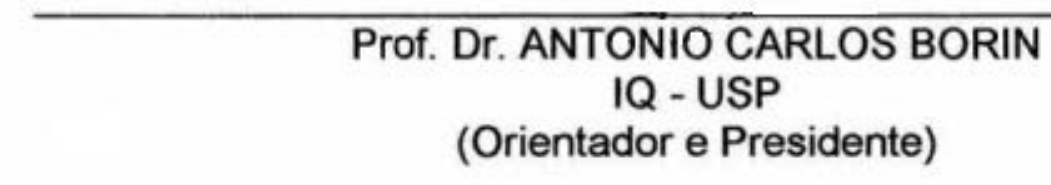

\begin{tabular}{c}
\hline Prof. Dr. HARRALD VICTOR LINNERTT \\
IQ - USP
\end{tabular}

Prof. Dr. NELSON HENRIQUE MORGON

IQ - UNICAMP

SÃO PAULO

17 DE ABRIL 2002. 


\section{Conteúdo}

I Introdução e Métodos Teóricos $\quad 9$

1 Introduçāo $\quad 10$

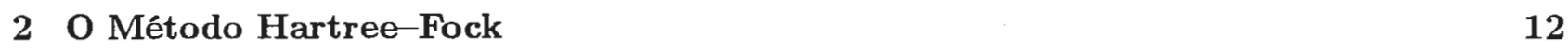

2.1 A Aproximação de Born-Oppenheimer . . . . . . . . . . . . . . . . . . 12

2.2 O Método Hartree-Fock . . . . . . . . . . . . . . . . . . . . . 14

2.3 Conjuntos de Bases Atômicas . . . . . . . . . . . . . . . . . . . 17

3 Métodos Correlacionados $\quad 22$

3.1 A Correlação Eletrônica . . . . . . . . . . . . . . . . . . . . . 22

3.2 O Método SCF Multiconfiguracional . . . . . . . . . . . . . . 23

3.3 O Método Interação de Configurações $\ldots \ldots \ldots \ldots$. . . . . . . . . . 26

$\begin{array}{ll}\text { II Resultados e Discussōes } & 30\end{array}$

4 Metodologia $\quad 32$

5 Resultados e Discussōes $\quad 34$

5.1 Caracterização dos Estados Eletrônicos . . . . . . . . . . . . . . . . 34

5.1 .1 O Estado Fundamental: $\mathrm{X}^{1} \Sigma^{+} \ldots \ldots \ldots \ldots \ldots$

5.1 .2 Os Estados $\mathrm{a}^{3} \Pi \mathrm{e}^{3} \Sigma^{+} \ldots \ldots \ldots \ldots \ldots \ldots \ldots$

5.1 .3 Os Estados $\mathrm{A}^{1} \Pi$ e $\mathrm{C}^{1} \Sigma^{+} \ldots \ldots \ldots \ldots \ldots \ldots \ldots \ldots$

5.1 .4 Os Estados $\mathrm{B}^{1} \Pi$ e $\mathrm{D}^{1} \Sigma^{+} \ldots \ldots \ldots \ldots \ldots \ldots \ldots \ldots$

5.1 .5 Análise dos Orbitais Moleculares . . . . . . . . . . . . . 51

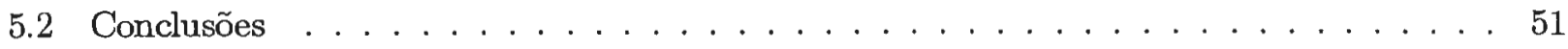

$\begin{array}{lll}\text { III Apêndice } & 57\end{array}$

$\begin{array}{ll}\text { A Curriculum Vitae } & \mathbf{5 8}\end{array}$

A.1 Formação Acadêmica . . . . . . . . . . . . . . . . . . . . . . 58

A.2 Cargos Ocupados . . . . . . . . . . . . . . . . . . . . . 59

A.3 Participação em Concurso Público . . . . . . . . . . . . . . . . . . . . 59

A.4 Comunicações em Congressos . . . . . . . . . . . . . . . . . . . . . 59 
A.5 Publicações 


\section{Lista de Tabelas}

5.1 Estados eletrônicos mais baixos energeticamente da molécula BeMg, a partir de dados atômicos, seus canais de dissociação e as respectivas energias de separação nos limites de dissociação. . . . . . . . . . . . . . . . . . . . . . . 35

5.2 Energias (em Hartree) dos estados eletrônicos da molécula BeMg. . . . . . . . . . . . 36

5.2. Continuação. Energias (em Hartree) dos estados eletrônicos da molécula BeMg. . . . . 37

5.3 Configurações eletrônicas mais importantes do BeMg. Os valores entre parenteses correspondem aos coeficientes das CSFs associadas às configurações eletrônicas. . . . 38

5.3 Continuação. Configurações eletrônicas mais importantes do BeMg. Os valores entre parenteses correspondem aos coeficientes das CSFs associadas às configurações

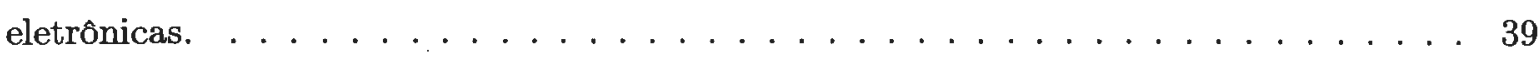

5.4 Espaçamentos vibracionais $\Delta_{v+1 / 2}\left(\mathrm{~cm}^{-1}\right)$ para os estados eletrônicos de menor energia da molécula BeMg. . . . . . . . . . . . . . . . . . . 44

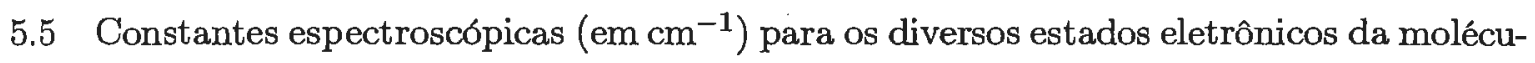
la BeMg. . . . . . . . . . . . . . . . . . . . . . . 44 


\section{Lista de Figuras}

5.1 Curvas de energia total para os estados singletos da molécula BeMg. . . . . . . . . . 41

5.2 Curvas de energia total para os estados tripletos da molécula BeMg. . . . . . . . . 42

5.3 Curva de energia total para o estado fundamental da molécula BeMg. . . . . . . . . 43

5.4 Seç̧ão transversal no plano $\mathrm{xz}$ dos orbital molecular $5 \sigma$, em torno da distância internuclear de equilíbrio. . . . . . . . . . . . . . . . . 45

5.5 Seç̧ão transversal no plano xz do orbital molecular $6 \sigma$, em torno da posição internu-

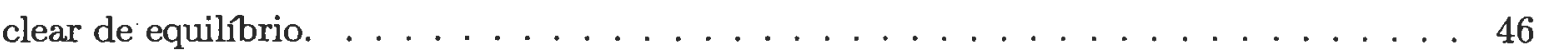

5.6 Seção transversal no plano xz do orbital molecular $7 \sigma$, em torno da posição internu-

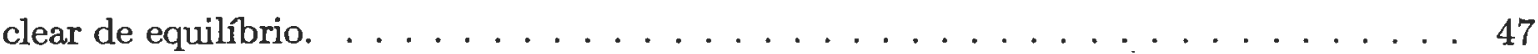

5.7 Seção transversal no plano xz do orbital molecular $2 \pi$ em torno da distância internuclear de equilíbrio. . . . . . . . . . . . . . . . . . . . . 49

5.8 Seç̧ão transversal no plano xz dos orbitais moleculares $5 \sigma, 6 \sigma, 7 \sigma$ e $2 \pi$ para diferentes distâncias internucleares. . . . . . . . . . . . . . . . . . . 52 


\section{Dedico este trabalho...}

A minha noiva e futura esposa, Kátia Mello, por estar ao meu lado desde o início, incentivando e apoiando incondicionalmente.

Aos meus pais, João e Josefa, por me apontar os rumos que deveria seguir. 


\section{Agradecimentos}

Ao longo deste trabalho, por várias vezes necessitei de conselhos e direcionamento. Nessas horas sempre pude recorrer ao meu orientador, Prof. Dr. Antonio Carlos Borin. Gostaria, portanto, de enfatizar um agradecimento especial pelo conhecimento, amizade, parceria, soluções e paciência.

Também contei com o apoio de vários amigos que fazem parte da minha vida profissional na SABESP. Mais ativamente o Pedro e a Érica que me incentivaram a começar e como já tinham trilhado o mesmo caminho, eventualmente me preveniam sobre algumas dificuladades.

Todos os funcionários do Instituto que me possibilitaram uma passagem tranquila sempre agindo com profissionalismo em todos os momentos que recorri a eles.

Ao pessoal do grupo de estudo, que me ajudou nas pequenas dúvidas que aconteciam e a apesar de todos terem uma carga pesada de trabalho, ainda conseguiam se distrair conversando sobre diversos assuntos. 


\section{Resumo}

O objetivo deste trabalho é descrever a estrutura eletrônica da espécie diatômica $\mathrm{BeMg}$, utilizando métodos $a b$ initio multiconfiguracionais. A primeira descrição teórica da espécie BeMg foi feita por Chiles e Dykstra em 1982, empregando os métodos SCF, CCD e CEPA. Naquele trabalho, os autores propuseram que a estrutura eletrônica do BeMg seria intermediária entre a apresentada pelas espécies $\mathrm{Be}_{2}$ e $\mathrm{Mg}_{2}$. Em 1994, Boldyrev et al. empregaram o método QCISD e conjuntos de bases atômicas do tipo $6-311+G^{*}$ para calcular a distância de equilíbrio do BeMg. Até onde sabemos, não existe nenhum outro estudo téorico, nem experimental, sobre esta espécie. Apesar dos esforços anteriores, devido à natureza dos átomos envolvidos, para descrever com maiores detalhes e precisão não somente o estado fundamental, mas também diferentes estados eletrônicos excitados, é necessário empregar métodos $a b$ initio multiconfiguracionais, fato explicitamente reconhecido por Boldyrev et al. em 1994.

No presente trabalho, as curvas de energia potencial para os estados eletrônicos energeticamente mais baixos, que correlacionam com os primeiros quatro canais de dissociação, da espécie $\mathrm{BeMg}$ foram descritas teoricamente empregando conjunto de bases atômicas do tipo cc-pVQZ e funções de onda do tipo CASSCF/MRCI. Todos os orbitais de valência, além de um conjunto adicional de funções de correlação do tipo $s$ e $p$ foram incluídos no espaço ativo. Diversas constantes espectroscópicas também foram calculadas para os estados eletrônicos selecionados. O estado fundamental foi caracterizado como de simetria ${ }^{1} \Sigma^{+}$e fracamente ligado $\left(D_{e}=0,05 \mathrm{eV}\right.$ ), possuindo consequentemente uma distância internuclear de equilíbrio relativamente longa $\left(3,30 \AA, \omega_{e}=44,2\right.$ $\mathrm{cm}^{-1}$ ). É interessante notar que nos trabalhos anteriores a distância internuclear de equilibrio do estado fundamental foi calculada com sendo 4,5 $\AA$ e 5,1 , por Chiles e Dykstra e Boldyrev et al., respectivamente. Os dois primeiros estados excitados são o a $a^{3} \Pi\left(\mathrm{R}_{e}=2,416 \AA, \mathrm{T}_{e}=11029 \mathrm{~cm}^{-1}\right)$ e o b $\Sigma^{+}\left(\mathrm{R}_{e}=2,578 \AA, \mathrm{T}_{e}=11058 \mathrm{~cm}^{-1}\right)$, ambos com energia de dissociação igual a $1,28 \mathrm{eV}$. 


\begin{abstract}
The first theoretical description of BeMg (using the SCF, CCD, and CEPA methods) was done by Chiles and Dykstra in 1982, when it was proposed that its electronic structure would be intermediate to $\mathrm{Be}_{2}$ and $\mathrm{Mg}_{2}$. In 1994, Boldyrev et al., using the QCISD method and $6-311+\mathrm{G}^{*}$ basis sets, presented other results on this diatomic species. To the best of our knowledge, there are no other study about this diatomic species. However, due to the nature of the atoms involved, it is necessary to employ more sophisticated theoretical methods to describe BeMg accurately.

In this study, potential energy curves for the lowest-lying electronic states correlating with the first four dissociation channels were determined using the cc-pVQZ basis sets and CASSCF/MRCI wave functions. All valence orbitals plus one set of $s$ and $p$ correlating functions were included in the active space. A whole set of spectroscopic constants completes the characterization of each state. In its ground state $\left(\mathrm{X}^{1} \Sigma^{+}\right) \mathrm{BeMg}$ is weakly bond $\left(\mathrm{D}_{e}=0.05 \mathrm{eV}\right)$, and consequently has a long internuclear equilibrium distance $\left(\mathrm{R}_{e}=3.30 \AA, \mathrm{w}_{e}=44.2 \mathrm{~cm}^{-1}\right)$. It is interesting to note that in previous theoretical works, the internuclear equilibrium distance was calculated to be around $4.5 \AA$ and 5.1 $\AA$. The first two excited states are an $\mathrm{a}^{3} \Pi\left(\mathrm{R}_{e}=2.416 \AA, \mathrm{T}_{e}=11029 \mathrm{~cm}^{-1}\right)$ and a b$\Sigma^{+}$ $\left(\mathrm{R}_{e}=2.578 \AA, \mathrm{T}_{e}=11058 \mathrm{~cm}^{-1}\right)$, with the same dissociation energy, $1.28 \mathrm{eV}$.
\end{abstract}




\section{Parte I}

\section{Introdução e Métodos Teóricos}




\section{Capítulo 1}

\section{Introdução}

Com o desenvolvimento de computadores capazes de armazenar e processar grandes quantidades de dados e de eficientes métodos mecânico-quânticos para cálculos de estrutura eletrônica e molecular, a química quântica tornou-se uma poderosa ferramenta de investigação científica [1]. Atualmente, somos capazes de descrever com excelente grau de precisão, o estado fundamental e diversos estados eletrônicos de espécies que variam de compostos diatômicos até modelos de proteínas, contendo ligações químicas fortes e fracas.

Empregando o método Interação de Configurações com excitações simples e duplas a partir de um conjunto de múltiplas referências, nosso grupo de pesquisa tem se destacado na descrição teórica de compostos diatômicos de berílio. Dentre as diversas espécies estudadas, fomos os primeiros a caracterizar detalhadamente as espécies $\mathrm{BeN}$ [2], $\mathrm{BeC}$ [3] e $\mathrm{BeB}$ [4]. Em continuidade a esta linha de pesquisa, o objetivo deste trabalho é descrever a estrutura a eletrônica da espécie diatômica BeMg, usando uma metodologia alternativa para a construção do espaço de referências. Nos trabalhos mencionados acima, o espaço de referências era gerado selecionado-se as configurações mais importantes para descrever os estados eletrônicos ao longo de toda a curva de energia potencial. Ao final desta seleção manual de configurações, feita com base no coeficiente de cada uma das configurações geradas, todas eram reunidas em um único conjunto que passa a ser empregado como espaço de referência para gerar as excitações simples e duplas. Nesta dissertação, o espaço de referências será gerado através do método Complete Active Space Self-Consistent Field (CASSCF). A grande vantagem deste método é que as configurações mais importantes são geradas automaticamente, com base num conjunto constituído pelos orbitais e elétrons mais importantes para a descrição do sistema.

A primeira descrição teórica da espécie BeMg foi feita por Chiles e Dykstra [5] em 1982, empregando os métodos SCF, CCD e CEPA. Naquele trabalho, os autores propuseram que a estrutura eletrônica do $\mathrm{BeMg}$ seria intermediária entre a apresentada pelas espécies $\mathrm{Be}_{2}$ e $\mathrm{Mg}_{2}$. Em 1994, Boldyrev et al. [6] empregaram o método QCISD e conjuntos de bases atômicas do tipo 6-311+G* para calcular a distância de equilíbrio do BeMg. Até onde sabemos, não existe nenhum outro estudo téorico, nem experimental, sobre esta espécie. Apesar dos esforços anteriores, devido à natureza dos átomos envolvidos, para descrever com maiores detalhes e precisão não somente o estado fundamental, mas também diferentes estados eletrônicos excitados, é necessário empregar métodos ab initio multiconfiguracionais, fato explicitamente reconhecido por Boldyrev et al. em 1994.

Existe, também, um interesse tecnológico neste tipo de composto. O grande potencial comercial 
de diodos que emitem lasers (azul e verde) e LEDs (light-emitting devices) tem aumentado muito o interesse por semicondutores do tipo II-VI e III-V. Atualmente, o tempo de vida de lasers verdes cuja base é ZnSe aumentou significativamente de alguns poucos segundos sobre condições pulsadas a $77 \mathrm{~K}$ para aproximadamente 100 horas. Por outro lado, cálculos teóricos indicaram que todos os calcogenetos de $\mathrm{Be}$ (Be chalcogenides) apresentam uma caráter iônico comparável ou menor ao da maioria dos semicondutores do tipo III-V. Lugauer et. al. [7] mostraram que a incorporação de Be aumenta em muito a dureza dos retículos, sugerindo que os LEDs e lasers contendo berílio teriam um tempo de vida maior do que os dispositivos padrões baseados em ZnSe. Um dos compostos estudados por Lugauer et al. é o (BeMg)Te.

Nos próximos capítulos, apresentaremos um breve resumo dos métodos teóricos empregados em nosso trabalho. Maiores detalhes poderão ser encontrados nas referências bibliográficas citadas ao longo do texto. 


\section{Capítulo 2}

\section{O Método Hartree-Fock}

O objetivo dos métodos quânticos computacionais é fornecer a melhor solução possível para a equação de Schrödinger independente do tempo

$$
\widehat{H} \Psi=E \Psi
$$

a partir da qual obtemos as funções de onda $(\Psi)$ e os valores de energia $(E)$ para os diversos estados eletrônicos. Sabemos que para os sistemas moleculares que estamos interessados em estudar, essa equação não possui solução analítica e, conforme mencionamos anteriormente, existem diversos métodos computacionais que permitem obter diversos tipos de soluções aproximadas. A seguir, descreveremos brevemente o método mais simples, conhecido como o Método Hartree-Fock (HF). Para uma discussão mais detalhada sobre os tópicos apresentados abaixo, sugerimos a leitura de Szabo e Ostlund [8], Almlöf [9] e Jensen [10].

\subsection{A Aproximação de Born-Oppenheimer}

O operador Hamiltoniano $(\widehat{H})$, não relativístico ${ }^{1}$, para a energia total de um sistema contendo $\mathrm{N}$ elétrons e $\mathrm{M}$ núcleos é dado por (em unidades atômicas):

$$
\begin{aligned}
& \widehat{H}=-\sum_{i}^{N} \frac{1}{2} \nabla_{i}^{2}-\sum_{A}^{M} \sum_{i}^{N} \frac{Z_{A}}{r_{i A}}+\sum_{i>j}^{N} \frac{1}{r_{i j}}-\sum_{A}^{M} \frac{1}{2 M_{A}} \nabla_{A}^{2}+\sum_{A>B}^{M} \frac{Z_{A} Z_{B}}{R_{A B}} \\
& \widehat{H}=\mathbf{T}_{e}+\mathbf{V}_{e N}+\mathbf{V}_{e e}+\mathbf{T}_{N}+\mathbf{V}_{N N}
\end{aligned}
$$

cada um dos termos representando, respectivamente,

- operador de energia cinética dos elétrons $\left(\mathbf{T}_{e}\right)$,

- operador de energia potencial de atração elétron-núcleo $\left(\mathbf{V}_{e N}\right)$,

- operador de potencial de repulsão intereletrônica $\left(\mathbf{V}_{e e}\right)$,

\footnotetext{
${ }^{1}$ Os efeitos relativísticos podem ser desprezados para os elementos dos três primeiros períodos da tabela periódica. Note, também, que estamos desprezando termos dependentes do spin eletrônico, como por exemplo os acoplamentos spin-orbita e spin-spin.
} 
- operador de energia cinética nuclear $\left(\mathbf{T}_{N}\right)$ e

- operador de potencial de repulsão nuclear $\left(\mathbf{V}_{N N}\right)$.

Se tentarmos resolver a Eq. 2.1 utilizando o operador Hamiltoniano $\widehat{H}$, obteremos um conjunto de funções de onda $(\Psi)$ dependentes das coordenadas eletrônicas (r) e nucleares $(\mathbf{R})$, sendo representadas por $\Psi(\mathbf{r}, \mathbf{R})$. Infelizmente, obter este conjunto de funções é uma tarefa difícil de ser executada na grande maioria de problemas de interesse químico.

Porém, existe uma maneira de torná-la mais fácil. Como sabemos, os núcleos são muito mais pesados do que os elétrons e, portanto, podem ser mantidos fixos ( $\mathbf{R}$ é constante) enquanto os elétrons movimentam-se rapidamente em torno deles. Em outras palavras, na maioria dos átomos e moléculas, a velocidade com que os elétrons se movimentam em torno dos núcleos é muito maior do que a velocidade do movimento nuclear mais rápido (movimento vibracional); portanto, os elétrons podem se rearranjar instantanemente os movimentos dos núcleos, que são muito mais lentos. Desta maneira, os movimentos eletrônicos e nucleares podem ser separados. Ou seja, na maioria dos problemas de interesse químico, a solução pode ser facilitada aproximando-se a função de onda exata $\Psi(\mathbf{r}, \mathbf{R})$ por um produto de duas funções, sendo que uma depende unicamente das coordenadas nucleares $(\chi(\mathbf{R}))$ e a outra depende das coordenadas eletrônicas, além de uma dependência paramétrica das coordenadas nucleares $(\psi(\mathbf{r} ; \mathbf{R}))$.

$$
\Psi(\mathbf{r}, \mathbf{R})=\psi(\mathbf{r} ; \mathbf{R}) \chi(\mathbf{R})
$$

Esta aproximação é conhecida como aproximação de Born-Oppenheimer.

Como estamos fixando a geometria nuclear, o termo corresponde à energia potencial de repulsão nuclear $\left(\mathbf{V}_{N N}\right)$ passa a ser constante e a energia cinética nuclear $\left(\mathbf{T}_{N}\right)$ desprezível. Portanto, podemos reescrever a Eq.2.2a como:

$$
\widehat{H}_{e}=-\sum_{i}^{N} \frac{1}{2} \nabla_{i}^{2}-\sum_{A}^{M} \sum_{i}^{N} \frac{Z_{A}}{r_{i A}}+\sum_{i>j}^{N} \frac{1}{r_{i j}},
$$

obtendo o operador Hamiltoniano puramente eletrônico $\left(\widehat{H}_{e}\right)$, no qual todos os termos possuem os mesmos significados atribuídos anteriormente.

A equação de Schrödinger puramente eletrônica é dada por:

$$
\left(\mathbf{T}_{e}+\mathbf{V}_{e N}+\mathbf{V}_{e e}\right) \psi(\mathbf{r} ; \mathbf{R})=E_{e l}(\mathbf{R}) \psi(\mathbf{r} ; \mathbf{R})
$$

sendo que tanto a energia eletrônica $\left(E_{e l}(\mathbf{R})\right)$ como a função de onda eletrônica $(\psi(\mathbf{r} ; \mathbf{R}))$ dependem parametricamente das coordenadas nucleares (R.), conforme está explicitamente indicado. Substituindo a Eq. (2.3) na Eq. (2.1) e usando a Eq. (2.5), obtemos:

$$
\left(\mathbf{T}_{N}+\mathbf{V}_{N N}+E_{e l}\right) \psi(\mathbf{r} ; \mathbf{R}) \chi(\mathbf{R})=E \psi(\mathbf{r} ; \mathbf{R}) \chi(\mathbf{R})
$$

Com R um parâmetro (aproximação de Born-Oppenheimer é válida),

$$
\left(\mathbf{T}_{N}+\mathbf{V}\right) \chi(\mathbf{R})=E \chi(\mathbf{R})
$$


sendo

$$
\mathbf{V}=\mathbf{V}_{N N}+E_{e l}
$$

conhecido como superficie de energia potencial eletrônica, que determina o movimento nuclear. Em outras palavras, dentro da aproximação de Born-Oppenheimer os núcleos estão se movendo em uma superfície de energia potencial que é a solução da equação de Schrödinger eletrônica (Eq. 2.5). Da maneira como estamos trabalhando, a superfície de energia potencial é independente das massas nucleares (isto é, é a mesma para moléculas contendo isótopos). Como podemos concluir, assumindo que a aproximação de Born-Oppenheimer seja válida, o problema se reduz a resolver a equação eletrônica de Schrödinger para um conjunto de geometrias nucleares, obtendo assim, a superfíce de energia potencial eletrônica. Com isso, podemos resolver a Eq. (2.7) e obter informações sobre os níveis vibracionais e rotacionais nucleares, as quais podem ser empregadas para se obter informações correspondentes às técnicas espectroscópicas correlatas.

\subsection{O Método Hartree-Fock}

A próxima etapa é resolver a equação eletrônica de Schrödinger. Analisando o operador Hamiltoniano puramente eletrônico (Eq. 2.4) notamos a presença do termo que representa a energia potencial de repulsão internuclear $\left(\mathbf{V}_{e e}\right)$, que correlaciona a posição instantânea de dois elétrons quaisquer (ou seja, de pares de elétrons). Devido a este termo, a solução exata da equação eletrônica de Schrödinger só é possível para sistemas moleculares contendo um único elétron, pois neste caso o termo $\mathbf{V}_{e e}$ é nulo, sendo o exemplo mais simples o sistema $\mathrm{H}_{2}^{+}$. Para sistemas mais complexos, precisamos recorrer aos métodos numéricos.

Para simplificar nossa discussão inicial, vamos considerar que o sistema seja composto por $\mathrm{N}$ elétrons não interagentes (i.e. modelo das partículas independentes), cujo Hamiltoniano é descrito por $(\widehat{h}(i)$, Eq. 2.9) $\Psi$

$$
\widehat{h}(i)=-\frac{1}{2} \nabla_{i}^{2}+\sum_{A}^{M} \frac{1}{r_{i A}}
$$

Além disso, vamos supor que $\widehat{h}(i)$ tenha como conjunto de autofunções um conjunto de spin-orbitais $\chi_{i}(\mathbf{x})$, que são formados pelo produto de um orbital espacial $(\psi(r))$ e uma função de spin ( $\omega$, que pode ser $\alpha$ ou $\beta$ ) (Eq. 2.11), formando um conjunto ortonormal de funções (Eq. 2.12).

$$
\begin{aligned}
\widehat{h}(i) \chi_{j}\left(\mathbf{x}_{i}\right) & =\varepsilon_{j} \chi_{j}\left(\mathbf{x}_{i}\right) \\
\chi_{j}(\mathbf{x}) & =\psi_{a}(r) \omega \\
\delta_{i j} & =<\chi_{i}(\mathbf{x}) \mid \chi_{j}(\mathbf{x})>
\end{aligned}
$$

Como os elétrons são independentes, a autofunção do operador Hamiltoniano do sistema de $\mathrm{N}$ elétrons pode ser escrita como um produto de $n$ funções de um elétron, i.e. spin-orbitais,

$$
\Psi^{\mathrm{HP}}=\chi_{i}\left(\mathbf{x}_{1}\right) \chi_{j}\left(\mathbf{x}_{2}\right) \ldots \chi_{n}\left(\mathbf{x}_{N}\right),
$$


conhecido como produto de Hartree ( $\left.\Psi^{\mathrm{HP}}\right)$.

$\mathrm{Na}$ prática, o produto de Hartree não pode ser empregado para descrever um sistema contendo $\mathrm{N}$ elétrons, porque:

1. Os elétrons não são correlacionados. Isto é, a probabilidade de se encontrar um elétron em uma determinada posição no espaço é independente da posição dos outros elétrons;

2. A função de onda $\Psi^{\mathrm{HP}}$ não é antissimétrica, o que implica em assumir que os elétrons são distingứveis. Como sabemos, funções de onda eletrônicas devem ser antissimétricas.

Uma forma muito simples de se obter funções de onda antissimétricas é antissimetrizando o produto de Hartree,

$$
\Psi_{\mathrm{AS}}=\frac{1}{\sqrt{N !}} \sum_{\mathbf{P}} \varepsilon_{\mathbf{p}} \mathbf{P} \chi_{i}\left(\mathbf{x}_{1}\right) \chi_{j}\left(\mathbf{x}_{2}\right) \ldots \chi_{n}\left(\mathbf{x}_{N}\right)
$$

onde $\mathbf{P}$ é um operador de permutação que troca somente as coordenadas dos spin-orbitais; $\varepsilon_{\mathbf{p}}$ corresponde ao sinal da permutação ( +1 ou -1 , se o número de permutações necessárias para gerar a configuração for par ou ímpar, respectivamente). A forma mais tradicional para representar a função de onda $\Psi_{\text {AS }}$ é a de um determinantes de Slater:

$$
\begin{aligned}
\Psi_{\mathrm{AS}} & =\frac{1}{\sqrt{N !}}\left|\begin{array}{cccc}
\chi_{i}\left(\mathbf{x}_{1}\right) & \chi_{j}\left(\mathbf{x}_{1}\right) & \ldots & \chi_{n}\left(\mathbf{x}_{1}\right) \\
\chi_{i}\left(\mathbf{x}_{2}\right) & \chi_{j}\left(\mathbf{x}_{2}\right) & \ldots & \chi_{n}\left(\mathbf{x}_{2}\right) \\
\vdots & \vdots & \ddots & \vdots \\
\chi_{i}\left(\mathbf{x}_{N}\right) & \chi_{j}\left(\mathbf{x}_{N}\right) & \ldots & \chi_{n}\left(\mathbf{x}_{N}\right)
\end{array}\right| \\
& =\mid \chi_{i}\left(\mathbf{x}_{1}\right) \chi_{j}\left(\mathbf{x}_{2}\right) \ldots \chi_{n}\left(\mathbf{x}_{N}\right)>
\end{aligned}
$$

Desta maneira, satisfazemos o princípio da antissimetria, além de introduzirmos um certo tipo de correlação entre os elétrons. Para compreendermos melhor esse ponto, vamos analisar a densidade de probabilidade de se encontrar um elétron com coordenada $\mathbf{x}_{1}$ e outro com $\mathbf{x}_{2}$ :

$$
\varrho\left(\mathbf{x}_{1}, \mathbf{x}_{2}\right)=\frac{1}{N(N-1)} \sum_{k, l}\left[\left|\chi_{k}\left(\mathbf{x}_{1}\right)\right|^{2}\left|\chi_{l}\left(\mathbf{x}_{2}\right)\right|^{2}-\chi_{k}^{*}\left(\mathbf{x}_{1}\right) \chi_{k}\left(\mathbf{x}_{2}\right) \chi_{l}^{*}\left(\mathbf{x}_{2}\right) \chi_{l}^{*}\left(\mathbf{x}_{1}\right)\right] .
$$

Como os spin-orbitais são escritos na forma de um produto de uma função espacial e uma função de spin, no caso de (ver Eq. 2.17) $\chi_{k}$ e $\chi_{l}$ possuirem spins opostos o segundo termo se anula, e portanto elétrons com spins opostos ainda não estão correlacionados. No entanto, se os spins forem iguais os dois termos se cancelam, indicando que elétrons com mesmo spin tendem a ficar isolados quando $r_{1}=r_{2}$; isto é, não ocupam a mesma posição no espaço, o que correlaciona o movimento de elétrons com mesmo spin. Este tipo de correlação entre o movimento dos elétrons é conhecido como correlação de troca. A região do espaço em torno do elétron é conhecida como buraco de Fermi (Fermi hole), sendo que nesta região a probabilidade de se encontrar elétrons com spin paralelos é nula.

Supondo que a função de onda multieletrônica mais simples seja expressa como um determinante de Slater, podemos empregar o método variacional e obter a melhor função de onda que minimiza o valor de energia do sistema

$$
\begin{aligned}
& E_{0}=\left\langle\Psi\left|\widehat{H}_{e}\right| \Psi\right\rangle \\
& \text { BIE: IOTECA } \\
& \text { WS } \\
& \text { Ualversitás's de São Paulo }
\end{aligned}
$$


onde $\Psi$ é um único determinante de Slater normalizado e $\widehat{H}_{e}$ o Hamiltoniano eletrônico escrito na seguinte forma:

$$
\widehat{H}_{e}=\sum_{i}^{N} \widehat{h}(i)+\sum_{i>j} \frac{1}{r_{i j}}+\mathbf{V}_{N N}
$$

Como estamos trabalhando dentro da aproximação de Born-Oppenheimer, o operador $\mathbf{V}_{N N}$ é uma constante. Utilizando estes operadores na expressão para energia, obtemos a seguinte expressão para a energia de uma função de onda Hartree-Fock:

$$
\begin{aligned}
E_{0}= & \sum_{i}^{N}<\chi_{i}|\widehat{h}(i)| \chi_{i}> \\
& +\frac{1}{2} \sum_{i j}^{N}\left(<\chi_{j}|\mathbf{J}(i)| \chi_{j}>-<\chi_{j}|\mathbf{K}(i)| \chi_{j}>\right)+\mathbf{V}_{N N}
\end{aligned}
$$

ou de uma maneira mais compacta

$$
E_{0}=\sum_{i}^{N}<\chi_{i}|\widehat{h}(i)| \chi_{i}>+\sum_{i>j}<\chi_{i} \chi_{j} \| \chi_{i} \chi_{j}>+\mathbf{V}_{N N}
$$

com

$$
\sum_{i>j}<\chi_{i} \chi_{j}|| \chi_{i} \chi_{j}>=\frac{1}{2} \sum_{i j}^{N}\left(<\chi_{j}|\mathbf{J}(i)| \chi_{j}>-<\chi_{j}|\mathbf{K}(i)| \chi_{j}>\right)
$$

na qual $\mathbf{J}(i)$ é o operador de Coulomb

$$
\mathbf{J}(i)\left|\chi_{j}(2)>=<\chi_{i}(1)\right| \frac{1}{r_{12}}\left|\chi_{i}(1)>\right| \chi_{j}(2)>
$$

e $\mathbf{K}(i)$ o operador de troca

$$
\mathbf{K}(i)\left|\dot{\chi}_{j}(2)>=<\chi_{i}(1)\right| \frac{1}{r_{12}}\left|\chi_{j}(1)>\right| \chi_{i}(2)>
$$

É importante ressaltar que o operador $\mathbf{J}(i)$ representa a multiplicação de um elemento de matriz com o mesmo orbital dos dois lados; o operador $\mathbf{K}(i)$ troca as duas funções do lado direito do operador $1 / r_{12}$.

O objetivo, agora, é encontrar um conjunto de spin-orbitais que minimize a energia do sistema, mantendo-os ortornormais. Aplicando o método dos multiplicadores de Lagrange, obtemos a seguinte expressão para a variação da energia do sistema:

$$
\begin{aligned}
\delta E & =\sum_{i}^{N}\left\langle\delta \chi_{i}\left|\widehat{h}_{i}\right| \chi_{i}\right\rangle+\left\langle\chi_{i}\left|\widehat{h}_{i}\right| \delta \chi_{i}\right\rangle+\sum_{i j}^{N}\left(\left\langle\delta \chi_{i}\left|\mathbf{J}_{j}+\mathbf{K}_{j}\right| \chi_{i}\right\rangle+\left\langle\chi_{i}\left|\mathbf{J}_{j}+\mathbf{K}_{j}\right| \delta \chi_{i}\right\rangle\right) \\
& =\sum_{i}^{N}\left(\left\langle\delta \chi_{i}\left|\mathbf{F}_{i}\right| \chi_{i}\right\rangle+\left\langle\chi_{i}\left|\mathbf{F}_{i}\right| \delta \chi_{i}\right\rangle\right) \\
\mathbf{F}_{i} & =\mathbf{h}_{i}+\sum_{j}^{N}\left(\mathbf{J}_{j}-\mathbf{K}_{j}\right)
\end{aligned}
$$


onde $\mathbf{F}_{i}$ é o operador de Fock, que descreve a energia cinética de um elétron, a atração de um elétron por todos os núcleos e a repulsão entre todos os elétrons. O operador de Fock está associado ao processo de minimização da energia total (variação da energia total) e não é igual ao Hamiltoniano total do sistema. O conjunto final de equações pode ser escrito como:

$$
\mathbf{F}_{i} \chi_{i}=\sum_{j}^{N} \lambda_{i j} \chi_{j}
$$

que são conhecidas como equações Hartree-Fock. Nesta equações, $\lambda_{i j}$ são os multiplicadores de Lagrange. Se escolhermos trabalhar com a forma diagonal da matriz dos multiplicadores de Lagrange, isto é, escolhermos os $\lambda_{i j}$ de tal forma que $\lambda_{i j} \rightarrow 0(i \neq j)$ e $\lambda_{i i}=\varepsilon_{i}$, onde $\varepsilon_{i}$ representa as energias orbitais, então poderemos reescrever as equações de Hartree-Fock da seguinte maneira:

$$
\begin{aligned}
\mathbf{F}_{i} \chi_{i}^{\prime} & =\varepsilon_{i} \chi_{i}^{\prime} \\
\varepsilon_{i} & =\left\langle\chi_{i}^{\prime}\left|\mathbf{F}_{i}\right| \chi_{i}^{\prime}\right\rangle=h_{i}+\sum_{j}^{N}\left(J_{i j}-K_{i j}\right)
\end{aligned}
$$

Em outras palavras, podemos dizer que após uma transformação matemática apropriada, conseguimos gerar um novo conjunto de spin-orbitais $\left\{\chi_{i}^{\prime}\right\}$, que são conhecidos como spin-orbitais canonicos, que diagonaliza a matriz formada pelos multiplicadores de Lagrange $\left(\lambda_{i j}\right)$

\subsection{Conjuntos de Bases Atômicas}

Até então, as equações de Hartree-Fock mencionam um conjunto genérico de spin-orbitais $\left\{\chi_{i}\right\}^{2}$. No entanto existem duas possibilidades para definirmos a parte espacial dos spin-orbitais. Quando a parte espacial $\left(\psi_{i}(r)\right)$ dos spin-orbitais for a mesma, independentemente da função de spin ser $\alpha$ ou $\beta$, chamamos o conjunto $\left\{\chi_{i}\right\}$ de conjunto de spin-orbitais restritos. Ou seja,

$$
\chi_{i}(r, \omega)=\left\{\begin{array}{l}
\psi_{i}(r) \alpha(\omega) \\
\psi_{i}(r) \beta(\omega)
\end{array} .\right.
$$

A outra possibilidade seria usar funções espaciais diferentes para cada função de spin

$$
\chi_{i}(r, \omega)=\left\{\begin{array}{c}
\psi_{i}^{\alpha}(r) \alpha(\omega) \\
\psi_{i}^{\beta}(r) \beta(\omega)
\end{array},\right.
$$

ou seja, os elétrons com spin $\alpha$ são descritos por um conjunto de funções espaciais $\left\{\psi_{i}^{\alpha}(r)\right\}$, os com spin $\beta$ pelo conjunto $\left\{\psi_{i}^{\beta}(r)\right\}$ diferente do conjunto $\left\{\psi_{i}^{\alpha}(r)\right\}$. Este conjunto de spin-orbitais (2.32) é conhecido como conjunto não restrito. Para simplificar a nossa análise, vamos nos restringir ao conjunto de spin-orbitais restritos.

\footnotetext{
${ }^{2}$ Para simplificar a notação, a partir de agora usaremos a notação $\left\{\chi_{i}\right\}$ para representar os orbitais canônicos, com os quais iremos trabalhar a partir de então.
} 
Se integramos a parte de spin das equações (2.29) iremos obter a seguinte expressão:

$$
\mathbf{F}_{i} \phi_{i}=\varepsilon_{i} \phi_{i}
$$

Note que na equação (2.33) estamos lidando apenas com os orbitais espaciais.

Para sistemas atômicos e diatômicos, as equações de Hartree-Fock podem ser resolvidas numericamente, contornando-se a necessidade de conhecermos a forma dos spin-orbitais a priori. No entanto, para sistemas maiores, a solução numérica é inviável. Nestes casos, todos os cálculos são feitos escrevendo os orbitais como uma combinação linear de funções de um conjunto base, que é conhecido como conjunto de bases atômicas. Temos, então, a aproximação LCAO (linear combination of atomic orbitals), sendo os orbitais $\phi_{i}$ escritos da seguinte forma:

$$
\phi_{i}=\sum_{\alpha=1}^{n} c_{\alpha i} \varphi_{\alpha}
$$

onde $\phi_{i}$ corresponde ao $i$-ésimo orbital molecular, $c_{\alpha i}$ são coeficientes de combinações lineares, $\varphi_{\alpha}$ representa o $\alpha$-ésimo orbital atômico de um conjunto base contendo $n$ funções. Substituindo a Eq. 2.34 na Eq. 2.33, obteremos as equações de Roothaan-Hall [11, 12], que correspondem às equações de Hartree-Fock na base dos orbitais atômicos:

$$
\mathbf{F C}=\mathbf{S C} \epsilon
$$

na qual $\mathbf{F}$ é a matriz de Fock, $\mathbf{C}$ a matriz dos coeficientes lineares, $\mathbf{S}$ a matriz de recobrimento e $\boldsymbol{\epsilon}$ a matriz contendo as energias orbitais;

$$
\begin{aligned}
& F_{\alpha \beta}=\left\langle\varphi_{\alpha}|\mathbf{F}| \varphi_{\beta}\right\rangle \\
& S_{\alpha \beta}=\left\langle\varphi_{\alpha} \mid \varphi_{\beta}\right\rangle
\end{aligned}
$$

Como anteriormente, estas equações devem ser resolvidas iterativamente. No final, obteremos um conjunto de coeficientes (matriz $\mathbf{C}$ ) que serão usados para gerar os orbitais moleculares otimizados, de acordo com a Eq. 2.34, e consequientemente a melhor função de onda multieletrônica na forma de um único determinante de Slater.

A qualidade dos orbitais moleculares está diretamente associada a do conjunto de bases atômicas $\left\{\varphi_{\alpha}\right\}$ empregado no cálculo. Conforme o número de funções contidas neste conjunto aumenta, os orbitais moleculares serão melhor descritos (Eq. 2.34); no limite de um conjunto de base atômico completo (número infinito de funções base), seremos capazes de reproduzir o resultado Hartree-Fock numérico; este limite é conhecido como limite Hartree-Fock. Mesmo assim, não teremos a solução exata da equação de Schrödinger, mas a melhor função de onda na forma de um único determinante de Slater que pode ser obtida. Na prática, somos obrigados a utilizar conjuntos de bases atômicos finitos.

Inicialmente, o conjunto de funções base $\left\{\varphi_{\alpha}\right\}$ era composto por orbitais de Slater (STO, i.e. Slater type orbitals), que são funções com a seguinte caracterísctica:

$$
\varphi_{\alpha}=(\xi, n, l, m ; r, \theta, \phi)=N r^{n-1} e^{\xi r} Y_{l m}(\theta, \phi) \text {, }
$$


onde $N$ é uma constante de normalização, $\xi$ o chamado expoente, $r, \theta$ e $\phi$ são as coordenadas esféricas e $Y_{l m}(\theta, \phi)$ os harmônicos esféricos, que descrevem a parte angular do orbital $\varphi_{\alpha} ; n, l$ e $m$ representam o número quântico principal, momento angular e magnético, respectivamente.

Funções deste tipo, no entanto, não são adequadas para se calcular integrais moleculares envolvendo dois elétrons. Para contornar essa dificuldade técnica, passou-se a representar os STO por combinações lineares de funções gaussianas cartesianas (GTO, i.e. gaussian type orbitals), que são representadas da seguinte forma:

$$
g(\alpha, n, l, m ; x, y, z)=N e^{-\alpha r^{2}} x^{l} y^{m} z^{n}
$$

com $N$ sendo uma constante de normalização e $\alpha$ o expoente; $x, y$ e $z$ representam as coordenadas cartesianas. É importante salientar que, neste caso, $n, l$ e $m$ não são números quânticos, mas simplesmente os expoentes das coordenadas cartesianas.

As funções descritas pela Eq. 2.39 são conhecidas por funções gaussianas primitivas, uma vez que elas não representam, propriamente, os orbitais atômicos. As diferenças básicas entre os orbitais atômicos e as gaussianas primitivas são:

- o fator pré exponencial, $r^{n-1}$, dos orbitais atômicos não aparece nas gaussianas primitivas;

- ao invés de uma dependência linear em $r$ no fator exponencial, nas funções gaussianas primitivas a dependência é quadrática $\left(e^{-\alpha r^{2}}\right)$. Essa dependência com $r^{2}$ da parte exponencial torna as GTO inferiores às STO em pelo menos dois aspectos. Em regiões muito próximas ao núcleo, a derivada das funções GTO é igual a zero, enquanto que as das STO são descontínuas (i.e. apresentam um cusp, ou seja uma derivada descontínua); em regiões muito distantes do núcleo as funções GTO decrescem muito rapidamente, em comparação ao comportamento das funçães STO.

- a parte que representa o momento angular é simplesmente uma função de coordenadas cartesianas.

É interessante mencionar que o valor de $L,(L=l+m+n)$, pode ser visto como um número análogo ao número quântico do momento angular orbital dos átomos, permitindo identificar funções do tipo $s(L=0), p(L=1), d(L=2)$, etc. Porém, existe um problema as funções do tipo $d$, ou com $L$ superiores. Sabemos que existem apenas cinco funções linearmente independentes e ortogonais para representar os orbitais atômicos; porém existem seis gaussianas cartesianas possíveis! Se somarmos todas as seis possíveis

$$
3 d_{x^{2}}+3 d_{y^{2}}+3 d_{z^{2}}=N\left(x^{2}+y^{2}+z^{2}\right) e^{-\alpha r^{2}}=r^{2} e^{-\alpha r^{2}}=3 s
$$

estaremos gerando uma função $3 s$ adicional. Fato semelhante ocorre com funções com $L$ superiores. Obviamente, os resultados obtidos em cálculos que incluem estes contaminantes serão diferentes daqueles obtidos sem estas funções adicionais.

Para minimizar os problemas mencionados acima, emprega-se um grande número de funções GTO para representar um orbital atômico (STO). O conjunto de todas as funções GTO consideradas importantes para representar os orbitais atômicos é conhecido como conjunto GTO primitivo. Para 
reduzir o custo computacional, opta-se por construir conjuntos menores de GTO combinando-se linearmente as funções contidas no conjunto GTO primitivo. O novo conjunto de funções é conhecido como conjunto GTO contraído, que pode ser obtido a partir de duas abordagens distintas: (i) contração segmentada e (ii) contração geral.

No método da contração segmentada cada função primitiva participa de uma única contração, ao passo que na contração geral todas as primitivas participam de todas as contrações. As funções obtidas pela contração segmentada são as mais populares pois os programas mais comuns operam de maneira muito mais eficiente com este tipo de funções do que com as obtidas através da contração geral, que requer códigos mais complexos. Dentre os conjuntos de funções bases obtidas pelo método da contração segmentada, podemos dizer que os desenvolvidos por Pople e colaboradores são as mais empregadas [13-18].

Além destas funções atômicas contraídas, adiciona-se a estes conjuntos outros tipos de funções. Os mais comuns são as funções de polarização e as funções difusas. Funções de polarização são simplesmente funções que possuem um valor de $L$ maior do que aquele presente nos orbitais atômicos ocupados do átomo correspondente, por exemplo funções do tipo $p$ para o átomo de hidrogênio. Essas funções são importantes para reproduzir as ligações químicas. As funções difusas são GTO com expoentes pequenos, que decaem lentamente com a distância internuclear; são normalmente do tipo $s, p$ e $d$. Essas funções são necessárias para descrever corretamente o comportamento e ânions e de ligação químicas muito fracas.

Em 1973, Raffenetti [19] introduziu o conceito de contração geral, no qual as mesmas funções gaussianas primitivas podem aparecer em diversos conjuntos contraídos. Teoricamente, a contração geral apresenta algumas vantagens sobre a contração segmentada. Dentre elas destacamos que com a. contração geral é possível reproduzir melhor os orbitais atômicos, o que facilitaria a interpretação dos coeficientes dos orbitais moleculares; além disso, o desempenho destes conjuntos de bases atômicas têm um desempenho muito melhor em cálculos que incluem efeitos de correlação eletrônica. Apesar destas vantagens, somente por volta de 1987 os conjuntos de bases atômicas obtidas pelo método da contração geral passaram a ser empregados mais frequentemente, graças aos trabalhos pioneiros de Almlöf e Taylor [20-22]. Nestes trabalhos iniciais, os autores empregaram conjuntos de orbitais naturais atômicos (ANO, atomic natural orbitals) como funções primitivas, obtidos a partir de um cálculo correlacionado para o átomo isolado. Como os orbitais naturais atômicos são bons para descrever os átomos isoladamente, espera-se que eles também sejam bons para descrever os átomos em ambientes moleculares.

Porém, em alguns tipos de átomos (i.e., os metais de transição) encontrarmos diferentes estados atômicos quasi-degenerados (com energias muito próximas). Portanto, se esses orbitais forem otimizados para um determinado estado, ele não será bom para descrever os outros. Em outras situações, como em complexos inorgânicos, muitas vezes os átomos têm um grande caráter iônico. Assim, em ambientes moleculares, o desempenho das ANO obtidas a partir de cálculos atômicos não é o esperado. Para contornar esse problema, pesquisadores como Bauschlicher, Langhoff e Roos [2328], dentre outros, propuseram o uso de orbitais naturais obtidos a partir de uma média de estados eletrônicos e iônicos (positivos e negativos) para formar o conjunto de funções primitivas, ao invés de usar o conjunto formado pelos orbitais naturais atômicos. 
Existe, ainda, um terceiro conjunto de funções base obtidos pelo método da contração geral, que está sendo muito utilizado. Essas funções foram desenvolvidos por Dunning e colaboradores [29-31] e são conhecidos como correlation consistent. As bases atômicas do tipo ANO são obtidas a partir de um conjunto muito grande de funções primitivas, garantindo praticamente a convergência no limite do conjunto base. Devido ao grande número de primitivas, os cálculos que empregam as bases atômicas do tipo ANO são muito demorados. Dunning e colaboradores propuseram o uso de um conjunto de primitivas relativamente menor, sem comprometer o resultado final, baseando-se na idéia de recuperar a energia de correlação dos elétrons de valência. Ao contrário das bases ANO, todas as funções que tiverem aproximadamente a mesma contribuição para a correlação eletrônica serão incluídas na mesma função final contraída, independentemente do tipo do momento angular. Por isso o nome correlation consistent. A estes conjuntos de bases atômicas, também deve-se adicionar funções difusas [32], conforme sugerido por Dunning e colaboradores, e/ou funções com expoentes maiores (tigh functions) [33], necessárias para descrever os efeitos de correlação dos elétrons mais internos.

Para obter maiores informações sobre os diversos tipos de bases atômicas mencionados anteriormente, além das referências mencionadas acima, recomendamos as revisões de Dunning e Hay [34], Feller e Davidson [35,36], Almlöf [9] e o capítulo correspondente do livro de Szabo e Ostlund [8]. 


\section{Capítulo 3}

\section{Métodos Correlacionados}

\subsection{A Correlação Eletrônica}

O método Hartree-Fock contém três importantes aproximações: (i) supõem-se que a função de onda possa ser descrita por um único determinante de Slater; (ii) substitui-se a interação média. entre os elétrons pela interação de um elétron com o campo médio gerado pelos outros; (iii) o uso dos conjuntos de bases atômicas. Obviamente, essas aproximações implicam em sérias limitações, sendo uma delas a incapacidade de descrever corretamente a dissociação de algumas moléculas, quando empregamos o método restrito. Vamos ilustrar este aspecto considerarando o cálculo da energia de dissociação da molécula de $\mathrm{N}_{2}$, a partir dos valores de energia para o átomo de nitrogênio e da molécula $\mathrm{N}_{2}$ obtidos em nível Hartree-Fock. Neste nível de cálculo, a energia da molécula é -108,99 u.a. (95\% da energia não relativística total) e a do átomo de nitrogênio é -54, 40 u.a. (energia total, $-54,59$ u.a.). Portanto, o valor calculado para energia de ligação do $\mathrm{N}_{2}$ é de $5,17 \mathrm{eV}$, sendo que o valor experimental conhecido é de $9,9 \mathrm{eV}$. Esse exemplo simples mostra que é necessário irmos além do método Hartree-Fock para se obter valores relativos de energia com precisão. Além disso, ele ressalta que, apesar do método Hartree-Fock ser suficiente para fornecer valores absolutos de energia com grande precisão, isso não significa que os valores relativos de energia serão precisos. Ou seja, pequenos erros nos valores absolutos poderão causar grandes erros nos valores relativos. Para resolver os problemas inerentes ao método Hartree-Fock, é necessário empregar métodos que incluam correlação eletrónica.

Por ser um método variacional, a energia Hartree-Fock será sempre menos negativa que a energia exata do sistema. Em 1959, Löwdin [37] definiu energia de correlação como sendo a diferença entre a energia exata não relativística $(E)$ e a energia obtida pelo método Hartree-Fock $\left(E_{H F}\right)$

$$
E_{\text {corr }}=E-E_{H F} \text {. }
$$

Para compreendermos um pouco melhor a origem da energia de correlação, basta recordarmos que o método Hartree-Fock utiliza o modelo de partículas independentes. Ou seja, o método supõe que o movimento de um determinado elétron é independente da posição instantânea dos outros, representando a função de onda como um único produto antissimetrizado de spin-orbitais (Determinante de Slater). Isso indica que o movimento eletrônico não está correlacionado, ou seja, a correlação dinâmica está sendo negligenciada. Consequentemente, os elétrons estão muito próximos na aproximação 
Hartree-Fock e, portanto, o termo de repulsão elétron-elétron torna-se muito grande, gerando um valor $E_{H F}$ acima do valor de $E$. Esta parte da energia de correlação está associada ao termo $1 / r_{12}$, que descreve a repulsão eletrônica no Hamiltoniano. No entanto, como a função de onda descrita por um determinante de Slater obedece o princípio de exclusão de Pauli (e.g., dois elétrons com spin paralelos não podem ocupar o mesmo orbital molecular), um determinado tipo de correlação eletrônica, chamada de correlação de Fermi (ou de troca), que também está relacionada aos pares de elétrons, está incorporada no método Hartree-Fock. Matematicamente ela é descrita na expressão de energia pelo operador de troca. Consequentemente, neste nível de teoria, estão correlacionados os elétrons com spins paralelos, sendo a correlação entre elétrons de spins opostos completamente ignorada.

Outra situação na qual o método Hartree-Fock não fornece bons resultados é, obviamente, quando o estado eletrônico não pode ser bem descrito por um único determinante de Slater. Isto é, quanto existirem outros determinantes com energia muito próxima a do determinante Hartree-Fock. Um exemplo clássico desta situação ocorre para o átomo de berílio, no qual o orbital $2 p$ tem energia próxima à do $2 s$. Portanto, a configuração $1 s^{2} 2 p^{2}$ também é importante para descrever este átomo. Este exemplo serve também para entender a origem do outro nome utilizado quando se menciona efeitos de correlação não dinâmica: degenerate correlation ou near degenerate correlation.

Resumindo, devemos empregar métodos capazes de minimizar os erros inerentes ao método Hartree-Fock para podermos estudar propriedades de interesse químico. Incluir efeitos de correlação eletrônica é essencial. Ou seja, devemos utilizar métodos que recuperam a energia de correlação dinâmica e não dinâmica, principalmente se o objetivo for estudar curvas ou superfícies de energia potencial, diferentes estados eletrônicos de um sistema, energia de ionização e dissociação, etc.

Para finalizar esta introdução, gostaríamos de mencionar que muitas vezes não é nítida a divisão entre energia de correlação dinâmica e não-dinâmica. No entanto, essa classificação é prática para o desenvolvimento de métodos mais sofisticados que o Hartree-Fock. Além disso, de uma forma ou de outra, esses métodos nunca tratam somente de um único efeito de correlação.

\subsection{O Método SCF Multiconfiguracional}

Como vimos anteriormente, um dos tipos de correlação eletrônica não incorporada no método Hartree-Fock é conhecida como correlação não-dinâmica, cuja origem está na existência de diversas configurações com energias muito próximas a da configuração Hartree-Fock, tornando incorreta a descrição do sistema por um único determinante de Slater.

Outra situação na qual se observa este tipo de correlação, porém de uma maneira indireta, é no estudo de curvas de energia potencial, como por exemplo no caso da molécula $\mathrm{H}_{2}$. Neste caso, os orbitais moleculares $\Psi_{i}(1,2)$ podem ser escritos como combinações lineares de funções atômicas $1 s_{A}$ e $1 s_{B}$ do átomo de hidrogênio

$$
\Psi_{i}(1,2)=N_{i}\left(1 s_{A} \pm 1 s_{B}\right)
$$

onde $N$ é uma constante de normalização, e a combinação positiva gera o orbital molecular ligante $\left(\Psi_{1}(1,2)\right)$, e a negativa o anti-ligante $\left(\Psi_{2}(1,2)\right)$. 
$\mathrm{Na}$ distância internuclear de equilíbrio a configuração eletrônica da molécula $\mathrm{H}_{2}$ é dada por $\Psi_{1}^{2}(1,2)$, ou seja, os dois elétrons ocupando o orbital ligante acoplados de maneira a formar um estado de spin singleto. Incluindo a função de spin correspondente, a função de onda pode ser escrita da seguinte forma (ignorando-se a constante de normalização):

$$
\Psi_{1}(1,2)=\left(1 s_{A}+1 s_{B}\right)(1)\left(1 s_{A}+1 s_{B}\right)(2)(\alpha(1) \beta(2)-\alpha(2) \beta(1))
$$

Como anteriormente, as funções $1 s_{a}$ e $1 s_{b}$ são as funções atômicas $1 s$ do átomo de hidrogênio, centradas nos átomos $A$ e $B$, respectivamente; a função $(\alpha(1) \beta(2)-\alpha(2) \beta(1))$ garante que o sistema está no estado singleto, correspondente ao estado fundamental da molécula $\mathrm{H}_{2}$.

Expandindo-se a função de onda $\Psi_{1}(1,2)$, temos:

$$
\begin{aligned}
\Psi_{1}(1,2)= & \left(1 s_{A}(1) 1 s_{A}(2)+1 s_{A}(1) 1 s_{B}(2)+\right. \\
& \left.1 s_{B}(1) 1 s_{A}(2)+1 s_{B}(1) 1 s_{B}(2)\right)(\alpha(1) \beta(2)-\alpha(2) \beta(1))
\end{aligned}
$$

Observa-se nesta expressão a presença de uma par de termos iônicos $\left(\Phi_{\text {ion }}(1,2)=1 s_{A}(1) 1 s_{A}(2)\right.$ e $\left.1 s_{B}(1) 1 s_{B}(2)\right)$ participando da função de onda total com o mesmo peso que os termos covalentes $\left(\Phi_{\text {cov }}(1,2)=1 s_{A}(1) 1 s_{B}(2)\right.$ e $\left.1 s_{B}(1) 1 s_{A}(2)\right)$.

O que aconteceria se tentássemos descrever a dissociação da molécula de $\mathrm{H}_{2}$ utilizando a função de onda RHF? Como todos os termos têm o mesmo peso, os iônicos continuariam contribuindo, gerando uma situação fisicamente irreal correspondentes à estruturas do tipo $\mathrm{H}^{+}$e $\mathrm{H}^{-}$.

Se analisarmos mais atentamente a função de onda expandida verificamos que é possível reescrevêla considerando a participação de cada termo

$$
\Psi_{1}(1,2)=C_{\text {ion }} \Phi_{\text {ion }}+C_{\text {cov }} \Phi_{\text {cov }}
$$

na qual $C_{\text {ion }}$ e $C_{\text {cov }}$ representam coeficientes variáveis. Novamente, as constantes de normalização foram omitidas. Empregando funções de onda deste tipo, e otimizando-se variacionalmente os coeficientes $\left(C_{\text {ion }}\right.$ e $\left.C_{\text {cov }}\right)$ e os orbitais moleculares, em torno da distancia de equilíbrio obteríamos $C_{\text {ion }}$ $\sim C_{\text {cov }}$, e no limite de dissociação teríamos $C_{\text {ion }} \rightarrow 0$, reproduzindo de maneira correta a dissociação da molécula de $\mathrm{H}_{2}$.

Este método de se obter funções utilizando-se combinações lineares de configurações eletrônicas para descrever os diversos estados eletrônicos é conhecido como Multiconfigurational Self Consistent Field Theory (MCSCF) [38].

No método MCSCF, os coeficientes das configurações eletrônicas e os orbitais moleculares são otimizados variacionalmente, demandando um esforço computacional muito maior do que no caso do * método Hartree-Fock. No entanto, se formos cuidadosos em aplicá-lo, poderemos reduzir o custo computacional sem comprometer a qualidade dos resultados. Queremos dizer que para os resultados serem bons, a escolha das configurações que serão empregadas na construção da função de onda deve ser feita com muito critério. Após a escolha destas configurações, elas são adaptadas à simetria espacial e de spin do problema, formando combinações lineares de determinantes de Slater conhecidas como configuration state functions (CSFs). São as CSFs que são empregadas para descrever a função de onda multiconfiguracional final. 
A construção destas configurações é feita, atualmente, por um processo automatizado, empregando-se o método CASSCF (Complete Active Space Self-Consistent Field) [39,40], desenvolvido por Roos e colaboradores. Neste método, assim como no MCSCF, as funções de onda são escritas na forma de combinações lineares de CSFs. No método CASSCF, o conjunto de orbitais moleculares é divido em (i) orbitais inativos, (ii) orbitais ativos e (iii) orbitais virtuais (ou secundários). Os orbitais inativos serão mantidos duplamente ocupados em todas as CSFs; além disso, os orbitais mais internos (carroço) poderão ser congelados na forma obtida durante um cálculo do tipo SCF. Os orbitais moleculares ativos serão aqueles construídos a partir de combinações lineares dos orbitais atômicos de valência; o número de ocupação destes orbitais moleculares ativos pode variar de um número próximo a zero até próximo a dois. Os outros orbitais formarão o espaço virtual, ou secundário, e sempre terão ocupação igual a zero. Como exemplo, vamos considerar a molécula de $\mathrm{C}_{2}$, cujas configurações eletrônicas podem ser geradas distribuindo-se os elétrons da seguinte maneira:

$$
\left(1 \sigma_{g}\right)^{2}\left(1 \sigma_{u}\right)^{2}\left\{2 \sigma_{g}, 2 \sigma_{u}, 3 \sigma_{g}, 3 \sigma_{u}, 1 \pi_{u x}, 1 \pi_{u y}, 1 \pi_{g x}, 1 \pi_{g y}\right\}^{8}
$$

Os orbitais mais internos dos átomos de carbono (1s) combinam-se para formar os orbitais moleculares $1 \sigma_{g}$ e $1 \sigma_{u}$. Portanto, podemos considerar que esses orbitais moleculares não são relevantes para descrever as propriedades de valência da molécula de $\mathrm{C}_{2} \mathrm{e}$, portanto, podemos mantê-los duplamente ocupados, formando o conjunto de orbitais do carroço, contendo quatro elétrons. Os orbitais atômicos de valência dos átomos de carbono $\left(2 s, 2 p_{x}, 2 p_{y}\right.$ e $\left.2 p_{z}\right)$ combinam-se linearmente para formar os orbitais moleculares $2 \sigma_{g}, 2 \sigma_{u}, 3 \sigma_{g}, 3 \sigma_{u}, 1 \pi_{u x}, 1 \pi_{u y}, 1 \pi_{g x}$ e $1 \pi_{g y}$. Como esses oito orbitais moleculares são formados a partir dos orbitais de valência dos átomos de carbono, eles devem ser incluídos no espaço ativo. Como quatro elétrons já foram atribuidos ao espaço inativo (os elétrons dos orbitais atômicos $1 s$ ), os outros oito elétrons serão considerados ativos e serão distribuídos entre os oitos orbitais moleculares ativos selecionados acima. A única restrição é que somente serão incluídas na nossa expansão aquelas ocupações cuja simetria espacial e de spin sejam a mesma do estado fundamental, que é o estado que desejamos estudar. Sabe-se que, no caso da molécula de $\mathrm{C}_{2}$, o estado fundamental é ${ }^{1} \Sigma_{g}^{+}$, sendo a configuração eletrônica representada por (não incluindo os elétrons do carroço):

$$
\ldots\left(2 \sigma_{g}\right)^{2}\left(2 \sigma_{u}\right)^{2}\left(1 \pi_{u}\right)^{4}
$$

Os elétrons no orbital $2 \sigma_{u}$ são essencialmente não ligantes, conferindo um caráter birradical à molécula. A configuração eletrônica

$$
\ldots\left(2 \sigma_{g}\right)^{2}\left(3 \sigma_{u}\right)^{2}\left(1 \pi_{u}\right)^{4}
$$

interage fortemente com a configuração anterior (3.7), proporcionando uma separação dos elétrons entre os dois átomos. É interessante mencionar que o peso relativo $\left(C_{3.7} / C_{3.8}\right)$ das configurações citadas acima é 0,19 , quanto a distância C-C for igual a 2,35 a.u. (valor experimental é 2.348 a.u.). Esse valor relativamente alto, ilustra a necessidade de incluirmos efeitos de correlação dinâmica para descrever sistemas que estão próximos da geometria de equilíbrio.

É importante ressaltar que os métodos MCSCF e CASSCF são boas opções para incluir na função de onda os efeitos de correlação eletrônica não dinâmica. Portanto, para obtermos os resultados com a precisão desejada é necessário, ainda, incluir os efeitos de correlação eletrônica dinâmica. 


\subsection{O Método Interação de Configurações}

Para incluir os efeitos de correlação dinâmica, empregamos o método interação de configurações (configuration interaction, $C I)[8,10,41,42]$. Uma função de onda do tipo CI é escrita como uma combinação linear de determinantes (configurações), cujos coeficientes serão determinados minimizandose a energia do sistema,

$$
\Psi=a_{0} \phi_{S C F}+\sum_{S} a_{S} \phi_{S}+\sum_{D} a_{D} \phi_{D}+\sum_{T} a_{T} \phi_{T}+\ldots=\sum_{i} a_{i} \phi_{i}
$$

onde cada um dos $\phi_{i}$ representa um conjunto de determinantes de Slater formados a partir de um conjunto de $n$ spin-orbitais $(\chi)$, que são construídos a partir de combinações lineares de orbitais atômicos $(\varphi)$ (Eq. 2.34); os índices $S, D$ e $T$, representam determinantes obtidos a partir de excitações simples, duplas e triplas, respectivamente, e $\varphi_{S C F}$ é a função de onda SCF. Se a expansão CI (Eq. 3.9) incluir todos os determinantes possíveis (excitando-se todos os elétrons), a função de onda será chamada de interação de configurações completa ( full ci, FCI). Isso é impossível de ser feito na prática, a menos que o sistema e o conjunto de bases atômicas sejam pequenos. Por isso, a expansão sempre será truncada em algum nivel de excitação. Os orbitais moleculares (spin-orbitais) normalmente são obtidos de um cálculo SCF ou MCSCF feitos anteriormente e serão mantidos desta forma. Ou seja, numa função de onda CI somente os coeficientes $a_{i}$ serão otimizados variacionalmente. Essa é a primeira diferença entre uma função de onda CI e uma MCSCF. O conjunto de orbitais moleculares também é subdividido, como no método MCSCF, porém em três grupos básicos, que incluem os orbitais do caroço, os inativos e os ativos, como no método MCSCF. Porém, neste caso, os ativos serão todos os orbitais de valência mais os virtuais; no método MCSCF os orbitais ativos englobam apenas os orbitais de valência, podendo ser ampliado dependendo do caso. Portanto, temos mais uma diferença entre o método MCSCF e CI.

A condição de normalização é dada por

$$
\sum_{i} c_{i}^{2}=1
$$

e os coeficientes $c_{i}$ determinados variacionalmente aplicando-se o método dos multiplicadores de Lagrange, que resulta no seguinte sistema de equações lineares

$$
\sum_{i}\left(H_{j i}-E S_{j i}\right) c_{i}=0
$$

sendo os valores de $E$ definidos a partir da equação secular

$$
\operatorname{det}\left(H_{j i}-E S_{j i}\right)=0
$$

sendo os elementos de matriz definidos da seguinte forma:

$$
\begin{aligned}
H_{j i} & =\left\langle\varphi_{j}|\mathbf{H}| \varphi_{i}\right\rangle \\
S_{j i} & =\left\langle\varphi_{j} \mid \varphi_{i}\right\rangle
\end{aligned}
$$

Como podemos observar, o método CI é simples, tanto do ponto de vista formal como conceitual. Além disso, se estivermos trabalhando com um conjunto completo de funções base, as Eqs. 3.11 e 
3.12 fornecerão o resultado exato. No entanto, na prática, somente conjuntos de bases finitos podem ser empregados e, mesmo assim, o número de determinantes envolvidos em um cálculo do tipo FCI torna essa abordagem inviável computacionalmente.

Portanto, a menos que estejamos trabalhando com sistemas muito simples, é necessário incluir somente alguns tipos de determinantes (excitações) na expansão CI (Eq. 3.9), gerando o que chamamos de expansão CI truncada (truncated CI expansion). O requisito fundamental para podermos trabalhar com segurança com uma expansão CI truncada é que ela seja capaz de recuperar grande parte da energia de correlação e ser suficientemente flexível para descrever uniformemente a estrutura eletrônica do sistema em toda a superfície de energia potencial.

Qual seria a expansão CI truncada mais simples? Como em muitos casos a configuração HartreeFock pode ser considerada como uma boa aproximação inicial para o sistema, vamos iniciar escolhendoa como estado de referência; isto é, nossa referência será um único determinante de Slater, conforme indicado na Eq. 3.9. Como somente determinantes que diferem em no máximo dois spin-orbitais possuem elementos de matriz (Eq. 3.13) diferentes de zero e são os que mais contribuem para correções na função de onda ordem zero, de acordo com teoria de perturbação, concluímos que a expansão CI trucada mais simples seria dada por

$$
\Psi_{S D}=a_{0} \varphi_{S C F}+\sum_{S} a_{S} \varphi_{S}+\sum_{D} a_{D} \varphi_{D}
$$

ou seja, aquela que inclui somente determinantes formados a partir de excitações simples ${ }^{1}$ e duplas da configuração Hartree-Fock. Essa função de onda é conhecida como função de onda CI simples e duplas, sendo representada pela sigla SDCI (singles and doubles CI).

Como o método SDCI acrescenta correções à função de referência, que é baseada na função de onda Hartree-Fock (o que pode ser considerado uma boa aproximação para sistemas em torno da posição de equilíbrio), ele não será capaz de fornecer resultados quantitativos para os casos nos quais a função de onda Hartree-Fock não for uma boa aproximação de ordem-zero. Ou seja, se a função de onda de ordem zero não for uma boa aproximação, não obteremos o resultado correto mesmo que correções importantes sejam feitas. Como já sabemos como descrever funções de onda contendo mais do que uma configuração através do método CASSCF, vamos acoplar esses dois métodos para contornar essa deficiência do método CISD.

Ao invés de usar como função de onda de ordem zero uma única configuração, passaremos a usar como espaço de referência mais do que uma configuração, gerando uma função de onda do tipo interação de configurações multiconfiguracional (multireference $C I$ ), MRCI, incluindo na expansão final todas as configurações contidas no espaço de referência, além daquelas geradas excitando-se elétrons a partir do conjunto de referência. Normalmente, somente serão incluídas excitações simples e duplas, sendo a função de onda final (MRSDCI) descrita da seguinte forma:

$$
\Psi_{M R S D C I}=\sum_{I} \Psi_{\mathrm{REF}}(I)=\sum_{I}\left\{C(I) \Phi(I)+\sum_{i x} C_{i}^{x}(I) \Phi_{i}^{x}(I)+\sum_{i j x y} C_{i j}^{x y}(I) \Phi_{i j}^{x y}(I)\right\}
$$

\footnotetext{
${ }^{1}$ Apesar dos elementos de matriz envolvendo a função de onda de ordem zero e os determinantes unicamente excitados serem nulos, o número destes determinantes é relativamente pequeno, eles são importantes para descrever algumas propriedades e interagem com os determinantes duplamente excitados. Por isso, eles são incluídos na função de onda CISD.
} 
onde a soma é feita sobre todas as configurações $(I)$, contidas no espaço de referência; a notação $\Phi_{i}^{x}(I)$ indica que um único elétron (i) foi excitado para o orbital $x$, a partir da configuração de referência $\Phi(I)$. Note que, um orbital que está vazio (virtual, ou externo) em um estado de referência pode estar ocupado (interno) em um outro estado de referência, o que implica em dificuldades adicionais na. implementação do método. Por outro lado, como a função de onda de referência contém configurações excitadas, a função de onda final irá conter configurações com nível de excitação maior do que dois, por exemplo excitações triplas, quádruplas, etc.

O ponto chave do método MRCI é a escolha das configurações que serão empregadas na construção do espaço de referência, que deve conter todas as configurações necessárias para descrever toda a superfície de energia potencial e estados eletrônicos envolvidos. Como vimos anteriormente, o método CASSCF é especialmente prático para esta finalidade. Assim, a primeira etapa de um cálculo do tipo MRCI é construir uma função de onda do tipo CASSCF. Em seguida, os orbitais e as configurações gerados serão empregados para a construção da função de onda MRSDCI; a função de onda de referência contém todas as funções CAS (geradas durante a etapa CASSCF) e as outras configurações serão geradas a partir de excitações simples e duplas deste espaço de referência. Portanto, a energia de correlação não dinâmica será recuperada durante a construção do espaço de referência e, em seguida, os efeitos de correlação dinâmica serão incorporados na função de onda através do método interação de configurações.

Desta maneira, o método MRSDCI é capaz de fornecer resultados extremamente precisos. Infelizmente, nem sempre poderemos empregar todos os orbitais de valência para construir o conjunto de configurações de referência. Portanto, em muitos casos, é necessário escolher cuidadosamente quais orbitais serão empregados, para garantir uma descrição equilibrada de todos os processos envolvidos. Por isso, assim como o método CASSCF, o MRSDCI também deve ser usado com muito critério.

Finalizando esta seção, vamos analisar um exemplo simples, com o intuito de verificarmos a forma na qual a correlação eletrônica dinâmica é incluída em funções do onda do tipo CI, estudando o caso de uma função de onda CI para descrever o estado fundamental do átomo de hélio. Vamos supor que essa função de onda aproximada fosse escrita da seguinte forma:

$$
\begin{aligned}
\Psi= & \sum_{i=1}^{4} C_{i} \phi_{i}= \\
& \sum_{i=1}^{4} C_{i} \psi_{i}(1) \psi_{i}(2)[\alpha(1) \beta(2)-\alpha(2) \beta(1)],
\end{aligned}
$$

sendo

$$
\begin{array}{cc}
\psi_{1}(\vec{r})=e^{-a r} & \psi_{2}(\vec{r})=x \psi_{1}(\vec{r}) \\
\psi_{3}(\vec{r})=y \psi_{1}(\vec{r}) & \psi_{4}(\vec{r})=z \psi_{1}\left(\vec{r}^{\prime}\right)
\end{array}
$$

e

$$
C_{2}=C_{3}=C_{4}
$$

Desta forma, obtemos:

$$
\Psi=\exp \left[-a\left(r_{1}-r_{2}\right)\right]\left[C_{1}+C_{2}\left(r_{1}^{2}+r_{2}^{2}+r_{12}^{2}\right) / 2\right][\alpha(1) \beta(2)-\alpha(2) \beta(1)] .
$$


Como podemos observar, a função de onda CI introduz indiretamente o termo $r_{12}$, o qual correlaciona o movimento dos dois elétrons. Assim podemos correlacionar a posição instantânea dos dois elétrons (correlação dinâmica), obviamente obteremos resultados superiores aos obtidos pelo método Hartree-Fock. 


\section{Parte II}

\section{Resultados e Discussões}


Nesta seção iremos apresentar os detalhes técnicos empregados na realização deste trabalho. Inicialmente, apresentaremos os conjuntos de bases atômicas empregados para descrever os átomos de berilio e magnésio. Em seguida, discutiremos a escolha do espaço ativo e a construção da função de onda de ordem zero, que foi empregada para a construção da função de onda MRCI.

Após a descrição destes detalhes técnicos, apresentaremos os resultados obtidos e discutiremos detalhadamente a natureza dos estados eletrônicos baseados na descrição dos orbitais moleculares. 


\section{Capítulo 4}

\section{Metodologia}

Quando ao conjunto de bases atômicas, no presente trabalho optamos por empregar as bases atômicas conhecidas por correlated consistent polarized valence basis set (cc-PVXZ, onde X representa o tipo de função $(X=D, T, Q$ ou 5 , representando, respectivamente, conjuntos dupla zeta, tripla zeta, quádrupla zeta e quíntupla zeta) desenvolvidos por Dunning e colaboradores $[29,30]$. Dentre os diversos conjuntos disponíveis, optamos por descrever os átomos de $\mathrm{Be}$ e $\mathrm{Mg}$ empregando conjuntos de bases atômicas cc-PVQZ. Estes conjuntos podem ser representados pela notação [5s $4 p 3 d 2 f 1 g$ ], indicando que para cada átomo foram utilizadas 5 funções contraídas do tipo $s, 4$ do tipo $p, 3$ do tipo $d, 2$ do tipo $f$ e uma $g$; o conjunto de funções contraídas foi obtido a partir de um conjunto de primitivas contendo $(12 s 6 p 3 d 2 f 1 g)$. De acordo com a definição deste tipo de conjunto de bases atômicas, todos os contaminantes foram removidos em todos os cálculos, sendo que o conjunto base final engloba um total de 114 funções contraídas.

As curvas de energia potencial e as funções momento de transição eletrônico foram calculadas empregando o método Interação de Configurações Multiconfiguracional (MRCI) [8,43, 44], com base em uma função de onda de ordem zero do tipo CASSCF (complete active space self-consistent field) $[39,45,46]$. Este tipo de cálculo é realizado em duas etapas, que foram realizadas empregandose o programa MOLPRO-98, desenvolvido por H. J. Werner, P. J. Knowles e colaboradores [47]. Incialmente, a função de onda de ordem zero do tipo CASSCF foi obtida, empregando-se como espaço ativo o conjunto de orbitais de valência $s$ e $p$ dos átomos de berílio e magnésio, com seus respectivos elétrons. Ou seja, o espaço ativo é composto por quatro elétrons, distribuídos entre os orbitais de valência $2 s(3 s)$ e $2 p(3 p)$ dos átomos de $\mathrm{Be} \mathrm{e} \mathrm{Mg}$, respectivamente. Os orbitais $1 s$ foram mantidos congelados e os $2 s$ e $2 p$ do magnésio mantidos duplamente ocupados em todos os cálculos. Completanto o conjunto de orbitais ativos, adicionamos um conjunto de orbitais de correlação do tipo $s$ e $p$, conforme recomendado por Martin [48]. Em seguida, a mesma função CASSCF descrita acima foi empregada como referência em um cálculo do tipo MRCI, no qual todas configurações adapatadas à simetria espacial e de spin (CSFs), obtidas a partir de excitações simples e duplas de todos os orbitais de valência, foram incluídas na função de onda final.

As funções de onda e energias vibracionais foram obtidas resolvendo-se numericamente a equação radial de Schrödinger para um conjunto de números quânticos vibracionais e rotacionais. As funções de energia potencial foram obtidas ajustando-se funções cúbicas splines ao pontos calculados. As constantes espectroscópicas foram determinadas a partir de um ajuste de mínimos quadrados envol- 
vendo os níveis ro-vibracionais calculados. A análise vibro-rotacional foi realizada com o auxílio do programa VIBROT, parte integrante do software MOLCAS 4.0 [49]. 


\section{Capítulo 5}

\section{Resultados e Discussões}

Nesta seção apresentaremos os resultados obtidos e faremos uma discussão detalhada para o estado fundamental, analisando a curva de energia potencial, as energias vibracionais e constantes espectroscópicas. Para concluirmos o trabalho, os outros estados serão analisados de maneira semelhante.

Conforme apresentaremos a seguir, os seis primeiros canais de dissociação da espécie BeMg foram considerados neste trabalho, resultando em dez estados eletrônicos. Destes dez estados eletrônicos, apenas os dois estados eletrônicos $\left(\mathrm{c}^{3} \Pi\right.$ e d $\left.{ }^{3} \Sigma^{+}\right)$que estão correlacionados com o terceiro canal de dissociação $\left(\mathrm{Be}\left({ }^{3} \mathrm{P}_{u}\right)+\mathrm{Mg}\left({ }^{1} \mathrm{~S}_{g}\right)\right)$ são repulsivos. O único estado eletrônico $\left({ }^{3} \Sigma^{+}\right)$que se correlaciona com o quinto canal de dissociação $\left(B e\left({ }^{1} S_{g}\right)+M g\left({ }^{3} S_{g}\right)\right)$ não será incluído neste trabalho, uma vez que cálculos preliminares indicaram que ele se encontra em uma região de energia muito superior a dos outros estados envolvidos.

\subsection{Caracterização dos Estados Eletrônicos}

Conforme apresentamos na Introdução, um único estado eletrônico $\left({ }^{1} \Sigma^{+}\right)$da espécie BeMg foi descrito na literatura, até o presente momento, tendo este sido identificado como o estado fundamental. Na ausência de outras informações teóricas, e mesmo experimentais, a identificação das simetrias e do número de estados eletrônicos que serão estudados é feita através das regras correlação de Wigner-Witmer [50], que podem ser resumidas da seguinte forma. Se $M_{L_{1}}$ e $M_{L_{2}}$ forem os números quânticos magnéticos correspondentes aos momentos angulares orbitais $\mathbf{L}_{1}$ e $\mathbf{L}_{2}$ dos átomos separados, respectivamente, obtemos da definição de $\Lambda$ (momento angular orbital total do estado eletrônico molecular) que

$$
\Lambda=\left|M_{L_{1}}+M_{L_{2}}\right|
$$

Além disso, temos também que o spin total da molécula é dado por:

$$
\mathbf{S}=\mathbf{S}_{1}+\mathbf{S}_{2}
$$

onde $\mathbf{S}_{1} \mathrm{e} \mathbf{S}_{2}$ são os spins dos átomos separados. Para os números quânticos correspondentes temos:

$$
S=S_{1}+S_{2}, S_{1}+S_{2}-1, \ldots,\left|S_{1}-S_{2}\right|
$$


Para o caso específico da espécie BeMg, os resultados da análise de correlação de Wigner-Witmer são apresentados na Tabela 5.1, onde também incluímos as energias de separação experimentias entre os cinco primeiros canais de dissociação, bem como os valores calculados neste trabalho para esta propriedade. Como indicativo da qualidade de nossos resultados, compararemos os valores teóricos de separação energética entre os diversos canais de dissociação com os valores obtidos experimentalmente [51]. Como podemos observar, com exceção dos dois canais energeticamente mais altos $\left(\mathrm{Be}\left({ }^{1} \mathrm{~S}_{g}\right)+\right.$ $\mathrm{Mg}\left({ }^{3} \mathrm{~S}_{g}\right)$ e $\left.\mathrm{Be}\left({ }^{1} \mathrm{P}_{u}\right)+\mathrm{Mg}\left({ }^{1} \mathrm{~S}_{g}\right)\right)$, a concordância entre os valores experimentais e aqueles por nós obtidos é muito boa.

Tabela 5.1: Estados eletrônicos mais baixos energeticamente da molécula BeMg, a partir de dados atômicos, seus canais de dissociação e as respectivas energias de separação nos limites de dissociação.

\begin{tabular}{lccc}
\hline Atomos Separados & $\begin{array}{c}\text { Estados } \\
\text { Moleculares }\end{array}$ & $\begin{array}{c}\Delta E(\mathrm{eV})^{\mathrm{a}} \\
\text { Experimental }\end{array}$ & $\begin{array}{c}\Delta E(\mathrm{eV})^{\mathrm{b}} \\
\text { Teórico }\end{array}$ \\
\hline $\mathrm{Be}\left({ }^{1} \mathrm{~S}_{g}\right)+\mathrm{Mg}\left({ }^{1} \mathrm{~S}_{g}\right)$ & ${ }^{1} \Sigma^{+}$ & 0,00 & 0,0 \\
$\mathrm{Be}\left({ }^{1} \mathrm{~S}_{g}\right)+\operatorname{Mg}\left({ }^{3} \mathrm{P}_{u}\right)$ & ${ }^{3} \Sigma^{+},{ }^{3} \Pi$ & 2,71 & $2,60 / 2,60$ \\
$\mathrm{Be}\left({ }^{3} \mathrm{P}_{u}\right)+\operatorname{Mg}\left({ }^{1} \mathrm{~S}_{g}\right)$ & ${ }^{3} \Sigma^{+},{ }^{3} \Pi$ & 2,72 & $2,74 / 2,74$ \\
$\mathrm{Be}\left({ }^{1} \mathrm{~S}_{g}\right)+\mathrm{Mg}\left({ }^{1} \mathrm{P}_{u}\right)$ & ${ }^{1} \Sigma^{+}, 1 \Pi$ & 4,34 & $4,31 / 4,32$ \\
$\operatorname{Be}\left({ }^{1} \mathrm{~S}_{g}\right)+\operatorname{Mg}\left({ }^{3} \mathrm{~S}_{g}\right)$ & ${ }^{3} \Sigma^{+}$ & 5,10 & 5,34 \\
$\mathrm{Be}\left({ }^{1} \mathrm{P}_{u}\right)+\operatorname{Mg}\left({ }^{1} \mathrm{~S}_{g}\right)$ & ${ }^{1} \Sigma^{+},{ }^{1} \Pi$ & 5,27 & $5,34 / 5,34$ \\
\hline \hline
\end{tabular}

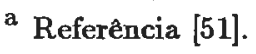

b Valores obtidos neste trabalho.

As energias e as configurações eletrônicas mais importantes dos dez estados eletrônicos calculados para a espécie BeMg estão compiladas nas Tabelas 5.2 (páginas 36 e 37) e 5.3 (páginas 38 e 39 ), respectivamente. Nos gráficos 5.1 e 5.2, apresentamos as curvas de energia potencial para todos os estados estudados. 
Tabela 5.2: Energias (em Hartree) dos estados eletrônicos da molécula BeMg.

\begin{tabular}{|c|c|c|c|c|c|}
\hline $\mathrm{R}\left(\mathrm{a}_{0}\right)$ & $\mathrm{X}^{1} \Sigma^{+}$ & $a^{3} \Pi$ & $\mathrm{b}^{3} \Sigma^{+}$ & $c^{3} \Pi$ & $\mathrm{d}^{3} \Sigma^{+}$ \\
\hline 3.250 & 0,124062 & 0,132505 & 0,088055 & 0,062652 & 0,009181 \\
\hline & 0,172164 & 0,169888 & & 02400 & \\
\hline 3.750 & 0,205976 & 3992 & 0,172619 & 0,127663 & \\
\hline 4.000 & 0,2290 & 0,208376 & & & \\
\hline 4.250 & 0,244 & 215846 & & 0,150253 & \\
\hline 4,500 & 0,254 & 499 & & & \\
\hline 4,750 & 0,2607 & 0,217886 & & 0,155022 & \\
\hline 5,000 & 0,2645 & 0,215176 & & & \\
\hline 5,250 & $0,2667 \varepsilon$ & 0,21 & & & \\
\hline 5,500 & $0,2679^{\prime}$ & 0,2 & & & \\
\hline 5,875 & 0,268713 & 0,199242 & & & \\
\hline 6,000 & 0,268802 & 0,196872 & 0,204721 & 0,168338 & \\
\hline 6,100 & 0,268839 & 0,195030 & 0,202961 & 0,168620 & 0,147247 \\
\hline 6,200 & 0,268853 & 0,193248 & 0,201205 & 0,168851 & 0,148912 \\
\hline 6,225 & 0,268854 & 0,192813 & 0,200768 & 0,168901 & 0,149316 \\
\hline 6,250 & 0,268854 & 0,192382 & 0,200332 & 0,168948 & 0,149716 \\
\hline 6,275 & 0,268853 & 0,191956 & 0,199897 & 0,168993 & 0,150107 \\
\hline 6,300 & 0,268851 & 0,191534 & 9464 & 9036 & \\
\hline 6,400 & 0,268840 & 0,189895 & 7748 & 9181 & 1960 \\
\hline 6,500 & 0,268815 & 0,188345 & 6059 & 295 & \\
\hline 6,600 & 0,268782 & 0,18 & 1423 & 376 & \\
\hline 6,750 & 0,268723 & 0,18 & 2068 & 447 & 326 \\
\hline 7,000 & 0,268611 & 0,181869 & 8442 & 0,169457 & 8800 \\
\hline 7,250 & 0,268492 & 0,179452 & 0,185234 & 9354 & 0,160816 \\
\hline 7,500 & 0,268371 & 0,177537 & 0,182466 & 0,169163 & 0,162437 \\
\hline 7,750 & 0,268252 & 0,176063 & 0,180131 & 0,168906 & 0,163718 \\
\hline 8,000 & 0,268137 & 0,174954 & 0,178205 & 0,168606 & 0,164707 \\
\hline 8,250 & 0,268028 & 0,174133 & 0,176653 & 0,168289 & 0,165449 \\
\hline 8,500 & 0,267925 & 0,173529 & 0,175429 & 0,167981 & 0,165982 \\
\hline 8,750 & 0,267830 & 0,173101 & 0,174507 & 0,167712 & 0,166351 \\
\hline 9,000 & 0,267742 & & & 0,167462 & 0,166574 \\
\hline 10,00 & 0,267465 & 0,172032 & 0,172379 & 0,166800 & 0,166713 \\
\hline 50,00 & 0,267017 & 0,171391 & 0,171433 & & 0,166198 \\
\hline
\end{tabular}


Tabela 5.2: Continuação. Energias (em Hartree) dos estados eletrônicos da molécula BeMg.

\begin{tabular}{cccccc}
\hline \hline $\mathrm{R}\left(\mathrm{a}_{0}\right)$ & $\mathrm{A}^{1} \Pi$ & $\mathrm{C}^{1} \Sigma^{+}$ & $\mathrm{e}^{3} \Sigma^{+}$ & $\mathrm{B}^{1} \Pi$ & $\mathrm{D}^{1} \Sigma^{+}$ \\
\hline 3,000 & 0,059787 & 0,046507 & & & 0,026833 \\
3,250 & 0,114337 & 0,091510 & 0,002606 & 0,054566 & 0,075608 \\
3.500 & 0,151326 & 0,119768 & 0,039988 & 0,096579 & 0,106288 \\
3.750 & 0,174944 & 0,135520 & 0,064580 & 0,123660 & 0,124266 \\
4.000 & 0,188730 & 0,142323 & 0,080604 & 0,139986 & 0,133707 \\
4.250 & 0,195477 & 0,142974 & 0,091476 & 0,148737 & 0,139485 \\
4,500 & 0,197284 & 0,145592 & 0,094479 & 0,152238 & 0,139291 \\
4,750 & 0,195700 & 0,149773 & 0,096996 & 0,152165 & 0,133384 \\
5,000 & 0,191850 & 0,151649 & 0,098648 & 0,149722 & 0,131686 \\
5,250 & 0,186548 & 0,151911 & 0,097616 & 0,145766 & 0,130210 \\
5,500 & 0,180372 & 0,151007 & 0,095291 & 0,140908 & 0,128357 \\
5,875 & 0,170337 & 0,148172 & 0,091125 & 0,132857 & 0,124751 \\
6,000 & 0,166926 & 0,146952 & 0,090016 & 0,130121 & 0,123364 \\
6,100 & 0,164203 & 0,145904 & 0,089432 & 0,127945 & 0,122202 \\
6,200 & 0,161498 & 0,144804 & 0,089177 & 0,125794 & 0,121000 \\
6,225 & 0,160826 & 0,144522 & 0,089160 & 0,125261 & 0,120694 \\
6,250 & 0,160155 & 0,144237 & 0,089161 & 0,124731 & 0,120386 \\
6,275 & 0,159487 & 0,143950 & 0,089176 & 0,124203 & 0,120076 \\
6,300 & 0,158820 & 0,143661 & 0,089205 & 0,123678 & 0,119764 \\
6,400 & 0,156181 & 0,142477 & 0,089428 & 0,121612 & 0,118503 \\
6,500 & 0,153577 & 0,141571 & 0,089824 & 0,119596 & 0,117215 \\
6,600 & 0,151021 & 0,140046 & 0,090170 & 0,117641 & 0,115910 \\
6,750 & 0,147273 & 0,138178 & 0,090684 & 0,114837 & 0,113928 \\
7,000 & 0,141368 & 0,135110 & 0,091360 & 0,110582 & 0,110598 \\
7,250 & 0,135895 & 0,132106 & 0,091671 & 0,106897 & 0,107278 \\
7,500 & 0,130929 & 0,129249 & 0,091570 & 0,103785 & 0,104009 \\
7,750 & 0,126527 & 0,126588 & 0,091079 & 0,101135 & 0,100825 \\
8,000 & 0,122738 & 0,124156 & 0,090251 & 0,098753 & 0,097751 \\
8,250 & 0,119588 & 0,121972 & 0,089159 & 0,096445 & 0,094811 \\
8,500 & 0,117063 & 0,120038 & 0,087876 & 0,094091 & 0,092020 \\
8,750 & 0,115100 & 0,118348 & 0,086506 & 0,091660 & 0,089391 \\
9,000 & 0,113605 & 0,116888 & 0,085039 & 0,089188 & 0,086934 \\
10,00 & 0,110470 & 0,112915 & 0,079436 & 0,080210 & 0,078912 \\
50,00 & 0,108114 & 0,108417 & 0,070608 & 0,070612 & 0,070620 \\
\hline \hline & & & & & \\
\hline
\end{tabular}


Tabela 5.3: Configurações eletrônicas mais importantes do BeMg. Os valores entre parenteses correspondem aos coeficientes das CSFs associadas às configurações eletrônicas.

\begin{tabular}{|c|c|c|c|c|c|}
\hline $\mathrm{R}$ & $\mathrm{X}^{1} \Sigma^{+}$ & $a^{3} \Pi$ & $\mathbf{b}^{3} \Sigma^{+}$ & $\mathrm{A}^{1} \Pi$ & $c^{3} \Pi$ \\
\hline \multirow[t]{2}{*}{3,00} & {$[c] 5 \sigma^{2} 6 \sigma^{2}(0.79)$} & {$[\mathrm{c}] 5 \sigma^{2} 6 \sigma^{1} 2 \pi^{1}(0.92)$} & {$[\mathrm{c}] 5 \sigma^{2} 6 \sigma^{1} 7 \sigma^{1}(0.94)$} & {$[\mathrm{c}] 5 \sigma^{2} 6 \sigma^{1} 2 \pi^{1}(0.91)$} & {$[\mathrm{c}] 5 \sigma^{2} 7 \sigma^{1} 2 \pi^{1}(0.79)$} \\
\hline & {$[c] 5 \sigma^{2} 2 \pi^{2}(0.31)$} & & & & {$[c] 5 \sigma^{1} 6 \sigma^{2} 2 \pi^{1}(0.79)$} \\
\hline \multirow[t]{2}{*}{4,00} & {$[\mathrm{c}] 5 \sigma^{2} 6 \sigma^{2}(0.88)$} & {$[\mathrm{c}] 5 \sigma^{2} 6 \sigma^{1} 2 \pi^{1}(0.91)$} & {$[\mathrm{c}] 5 \sigma^{2} 6 \sigma^{1} 7 \sigma^{1}(0.95)$} & {$[\mathrm{c}] 5 \sigma^{2} 6 \sigma^{1} 2 \pi^{1}(0.89)$} & {$[\mathrm{c}] 5 \sigma^{2} 7 \sigma^{1} 2 \pi^{1}(0.79)$} \\
\hline & {$[c] 5 \sigma^{2} 7 \sigma^{2}(0.25)$} & & & & {$[c] 5 \sigma^{1} 6 \sigma^{2} 2 \pi^{1}(0.79)$} \\
\hline \multirow[t]{2}{*}{5,00} & {$[c] 5 \sigma^{2} 6 \sigma^{2}(0.89)$} & {$[c] 5 \sigma^{2} 6 \sigma^{1} 2 \pi^{1}(0.90)$} & {$[c] 5 \sigma^{2} 6 \sigma^{1} 7 \sigma^{1}(0.93)$} & {$[\mathrm{c}] 5 \sigma^{2} 6 \sigma^{1} 2 \pi^{1}(0.87)$} & {$[\mathrm{c}] 5 \sigma^{2} 7 \sigma^{1} 2 \pi^{1}(0.79)$} \\
\hline & {$[\mathrm{c}] 5 \sigma^{2} 7 \sigma^{2}(0.21)$} & & & & {$[\mathrm{c}] 5 \sigma^{2} 7 \sigma^{1} 2 \pi^{1}(0.79)$} \\
\hline \multirow[t]{2}{*}{6,25} & {$[\mathrm{c}] 5 \sigma^{2} 6 \sigma^{2}(0.89)$} & {$[\mathrm{c}] 5 \sigma^{2} 6 \sigma^{1} 2 \pi^{1}(0.89)$} & {$[\mathrm{c}] 5 \sigma^{2} 6 \sigma^{1} 7 \sigma^{1}(0.91)$} & {$[\mathrm{c}] 5 \sigma^{2} 6 \sigma^{1} 2 \pi^{1}(0.83)$} & {$[\mathrm{c}] 5 \sigma^{2} 6 \sigma^{1} 3 \pi^{1}(0.79)$} \\
\hline & & {$[c] 5 \sigma^{1} 6 \sigma^{2} 3 \pi^{1}(0.29)$} & & {$[c] 5 \sigma^{2} 6 \sigma^{1} 3 \pi^{1}(0.26)$} & {$[\mathrm{c}] 5 \sigma^{1} 6 \sigma^{2} 2 \pi^{1}(0.79)$} \\
\hline \multirow[t]{2}{*}{7,00} & {$[c] 5 \sigma^{2} 6 \sigma^{2}(0.90)$} & {$[\mathrm{c}] 5 \sigma^{2} 6 \sigma^{1} 2 \pi^{1}(0.84)$} & [c] $5 \sigma^{2} 6 \sigma^{1} 7 \sigma^{1}(0.89)$ & {$[\mathrm{c}] 5 \sigma^{2} 6 \sigma^{1} 2 \pi^{1}(0.82)$} & {$[c] 5 \sigma^{2} 6 \sigma^{1} 3 \pi^{1}(0.83)$} \\
\hline & & {$[c] 5 \sigma^{1} 6 \sigma^{2} 3 \pi^{1}(0.40)$} & {$[\mathrm{c}] 5 \sigma^{1} 6 \sigma^{2} 8 \sigma^{1}(0.26)$} & {$[c] 5 \sigma^{2} 6 \sigma^{1} 3 \pi^{1}(0.26)$} & {$[c] 5 \sigma^{2} 6 \sigma^{1} 2 \pi^{1}(0.83)$} \\
\hline \multirow[t]{3}{*}{9,00} & {$[\mathrm{c}] 5 \sigma^{2} 6 \sigma^{2}(0.90)$} & {$[c] 5 \sigma^{2} 6 \sigma^{1} 2 \pi^{1}(0.84)$} & {$[\mathrm{c}] 5 \sigma^{2} 6 \sigma^{1} 7 \sigma^{1}(0.68)$} & [c] $5 \sigma^{2} 6 \sigma^{1} 2 \pi^{1}(0.87)$ & {$[\mathrm{c}] 5 \sigma^{2} 6 \sigma^{1} 3 \pi^{1}(0.83)$} \\
\hline & & {$[\mathrm{c}] 5 \sigma^{1} 6 \sigma^{2} 3 \pi^{1}(0.39)$} & {$[\mathrm{c}] 5 \sigma^{1} 6 \sigma^{2} 8 \sigma^{1}(0.46)$} & & [c] $5 \sigma^{1} 6 \sigma^{2} 2 \pi^{1}(0.83)$ \\
\hline & & & {$[\mathrm{c}] 5 \sigma^{2} 6 \sigma^{1} 8 \sigma^{1}(0.45)$} & & \\
\hline \multirow[t]{3}{*}{11,0} & {$[\mathrm{c}] 5 \sigma^{2} 6 \sigma^{2}(0.90)$} & {$[\mathrm{c}] 5 \sigma^{2} 6 \sigma^{1} 2 \pi^{1}(0.76)$} & {$[\mathrm{c}] 5 \sigma^{2} 6 \sigma^{1} 8 \sigma^{1}(0.75)$} & {$[\mathrm{c}] 5 \sigma^{2} 6 \sigma^{1} 2 \pi^{1}(0.87)$} & {$[\mathrm{c}] 5 \sigma^{1} 6 \sigma^{2} 2 \pi^{1}(0.83)$} \\
\hline & & {$[\mathrm{c}] 5 \sigma^{1} 6 \sigma^{2} 3 \pi^{1}(0.48)$} & {$[c] 5 \sigma^{2} 6 \sigma^{1} 7 \sigma^{1}(0.49)$} & & {$[\mathrm{c}] 5 \sigma^{2} 6 \sigma^{1} 3 \pi^{1}(0.83)$} \\
\hline & & & & & [c] $5 \sigma^{2} 6 \sigma^{1} 2 \pi^{1}(0.83)$ \\
\hline
\end{tabular}

$[c]=1 \sigma^{2} 2 \sigma^{2} 3 \sigma^{2} 4 \pi^{4} 4 \sigma^{2}$ 
Tabela 5.3: Continuação. Configurações eletrônicas mais importantes do BeMg. Os valores entre parenteses correspondem aos coeficientes das CSFs associadas às configurações eletrônicas.

\begin{tabular}{clllll}
\hline \hline $\mathrm{R}$ & $\mathrm{d}^{3} \Sigma^{+}$ & $\mathrm{B}^{1} \Pi$ & $\mathrm{C}^{1} \Sigma^{+}$ & $\mathrm{D}^{1} \Pi$ & $\mathrm{e}$ \\
\hline 3,00 & {$[\mathrm{c}] 5 \sigma^{2} 2 \pi^{1} 3 \pi^{1}(0.53)$} & {$[\mathrm{c}] 5 \sigma^{2} 7 \sigma^{1} 2 \pi^{1}(0.76)$} & {$[\mathrm{c}] 5 \sigma^{2} 2 \pi^{2}(0.66)$} & {$[\mathrm{c}] 5 \sigma^{2} 2 \pi^{2}(0.57)$} & {$[\mathrm{c}] 5 \sigma^{2} 2 \pi^{1} 3 \pi^{1}(0.61)$} \\
& {$[\mathrm{c}] 5 \sigma^{1} 6 \sigma^{1} 2 \pi^{2}(0.40)$} & {$[\mathrm{c}] 5 \sigma^{2} 8 \sigma^{1} 2 \pi^{1}(0.35)$} & & {$[\mathrm{c}] 5 \sigma^{2} 6 \sigma^{2}(0.39)$} & {$[\mathrm{c}] 5 \sigma^{1} 6 \sigma^{1} 2 \pi^{2}(0.27)$} \\
& & {$[\mathrm{c}] 5 \sigma^{2} 6 \sigma^{1} 3 \pi^{1}(0.30)$} & & \\
& & {$[\mathrm{c}] 5 \sigma^{2} 6 \sigma^{2} 2 \pi^{1}(0.20)$} & & & \\
& & & & & \\
4,00 & {$[\mathrm{c}] 5 \sigma^{2} 6 \sigma^{1} 9 \sigma^{1}(0.60)$} & {$[\mathrm{c}] 5 \sigma^{2} 7 \sigma^{1} 2 \pi^{1}(0.75)$} & {$[\mathrm{c}] 5 \sigma^{2} 2 \pi^{2}(0.58)$} & {$[\mathrm{c}] 5 \sigma^{2} 2 \pi^{2}(0.65)$} & {$[\mathrm{c}] 5 \sigma^{2} 2 \pi^{1} 3 \pi^{1}(0.58)$} \\
& {$[\mathrm{c}] 5 \sigma^{2} 2 \pi^{1} 3 \pi^{1}(0.48)$} & {$[\mathrm{c}] 5 \sigma^{2} 6 \sigma^{1} 3 \pi^{1}(0.43)$} & {$[\mathrm{c}] 5 \sigma^{2} 7 \sigma^{2}(0.29)$} & & \\
& & {$[\mathrm{c}] 5 \sigma^{2} 6 \sigma^{2} 2 \pi^{1}(0.24)$} & & & \\
& & & & & \\
5,00 & {$[\mathrm{c}] 5 \sigma^{2} 6 \sigma^{1} 7 \sigma^{1}(0.80)$} & {$[\mathrm{c}] 5 \sigma^{2} 7 \sigma^{1} 2 \pi^{1}(0.66)$} & {$[\mathrm{c}] 5 \sigma^{2} 6 \sigma^{1} 7 \sigma^{1}(0.70)$} & {$[\mathrm{c}] 5 \sigma^{2} 6 \sigma^{1} 7 \pi^{2}(0.33)$} \\
& {$[\mathrm{c}] 5 \sigma^{2} 6 \sigma^{1} 8 \sigma^{1}(0.32)$} & {$[\mathrm{c}] 5 \sigma^{2} 6 \sigma^{1} 3 \pi^{1}(0.54)$} & {$[\mathrm{c}] 5 \sigma^{2} 7 \sigma^{2}(0.38)$} & {$[\mathrm{c}] 5 \sigma^{2} 7 \sigma^{2}(0.43)$} & {$[\mathrm{c}] 5 \sigma^{2} 6 \sigma^{2} 7 \sigma^{1}(0.33)$} \\
& & {$[\mathrm{c}] 5 \sigma^{2} 6 \sigma^{2} 2 \pi^{1}(0.29)$} & & {$[\mathrm{c}] 5 \sigma^{2} 6 \sigma^{1} 8 \sigma^{1}(0.82)$} \\
& & & & & \\
& & & & & \\
6,25 & {$[\mathrm{c}] 5 \sigma^{2} 6 \sigma^{1} 7 \sigma^{1}(0.82)$} & {$[\mathrm{c}] 5 \sigma^{2} 7 \sigma^{1} 2 \pi^{1}(0.55)$} & {$[\mathrm{c}] 5 \sigma^{2} 6 \sigma^{1} 7 \sigma^{1}(0.74)$} & {$[\mathrm{c}] 5 \sigma^{2} 7 \sigma^{2}(0.49)$} & {$[\mathrm{c}] 5 \sigma^{2} 6 \sigma^{1} 8 \sigma^{1}(0.65)$} \\
& {$[\mathrm{c}] 5 \sigma^{2} 6 \sigma^{1} 8 \sigma^{1}(0.38)$} & {$[\mathrm{c}] 5 \sigma^{2} 6 \sigma^{1} 3 \pi^{1}(0.56)$} & {$[\mathrm{c}] 5 \sigma^{2} 7 \sigma^{2}(0.33)$} & {$[\mathrm{c}] 5 \sigma^{2} 6 \sigma^{1} 7 \sigma^{1}(0.44)$} & {$[\mathrm{c}] 5 \sigma^{1} 6 \sigma^{2} 7 \sigma^{1}(0.31)$} \\
& & {$[\mathrm{c}] 5 \sigma^{1} 6 \sigma^{2} 3 \pi^{1}(0.40)$} & {$[\mathrm{c}] 5 \sigma^{1} 6 \sigma^{2} 7 \sigma^{1}(0.30)$} & {$[\mathrm{c}] 5 \sigma^{1} 6 \sigma^{2} 7 \sigma^{1}(0.39)$} & {$[\mathrm{c}] 5 \sigma^{1} 6 \sigma^{1} 7 \sigma^{2}(0.23)$} \\
& & {$[\mathrm{c}] 5 \sigma^{1} 6 \sigma^{2} 2 \pi^{1}(0.28)$} & & {$[\mathrm{c}] 5 \sigma^{2} 6 \sigma^{1} 8 \sigma^{1}(0.38)$} & \\
\hline \hline
\end{tabular}




\subsubsection{O Estado Fundamental: $\mathrm{X}^{1} \Sigma^{+}$}

Em concordância com os trabalhos anteriores, determinamos que o estado fundamental do $\mathrm{BeMg}$ é de simetria ${ }^{1} \Sigma^{+}$, com uma distância internuclear de equilíbrio igual a $6,234 \mathrm{a}_{0}(3,30 \AA)$, sendo o momento de dipolo calculado igual a 0,219 D. Neste ponto, é importante ressaltar que os trabalhos anteriores determinaram distâncias internucleares de equilíbrio muito maiores: 4,5 [5] e 5,1 $\AA$ [6]. O estado fundamental correlaciona-se com o primeiro canal de dissociação $\left(\mathrm{Be}\left({ }^{1} \mathrm{~S}_{g}\right)+\mathrm{Mg}\left({ }^{1} \mathrm{~S}_{g}\right)\right.$, ver Tabela 5.1, página 35), tendo energia de dissociação igual a $0,05 \mathrm{eV}(1,2 \mathrm{kcal} / \mathrm{mol})$. Apresentamos na Figura 5.3 uma visão ampliada da curva de energia potencial para o estado $\mathrm{X}^{1} \Sigma^{+}$, para que ele possa ser melhor visualizado.

Antes de prosseguirmos com a descrição da estrutura eletrônica do estado fundamental do $\mathrm{BeMg}$, é importante verificarmos se a estabilidade prevista não é um artefato do cálculo, proveniente de um erro de sobreposição de bases atômicas (basis set superposition error, BSSE), frequentemente encontrado quando calculamos pequenas diferenças de energia (i.e., complexos de van der Waals e pontes de hidrogênio). A origem deste problema está associado ao emprego de conjuntos finitos de bases atômicas centrados nos núcleos; isto é, as funções atômicas centradas em um núcleo podem compensar a deficiência da base atômica do outro núcleo, e vice-versa. Portanto, a energia dos átomos unidos estaria artificialmente mais negativa, superestimando a energia de ligação. Uma maneira óbvia para contornar este problema é empregar conjuntos de bases atômicas bastante extensos, de tal modo que o que acréscimo de outras funções não acarretaria nenhuma variação na energia do sistema. Porém, estamos limitados ao emprego de conjuntos finitos de bases atômicas e, apesar de estarmos empregando conjuntos extensos (cc-pVQZ), como o valor calculado para a energia de dissociação do BeMg é relativamente pequeno, decidimos verificar se não está ocorrendo erro de sobreposição de bases. Para isso, empregamos o método conhecido como counterpoise correction [52,53]. A energia de dissociação corrigida através deste método é igual àquela obtida sem a correção, o que indica que não há erro de sobreposição de base. Portanto, podemos concluir que na distância internuclear de $3,30 \AA$, os átomos unidos são mais estáveis que separados.

A partir da curva de energia potencial para o estado fundamental, apresentada na Tabela 5.2 (página 36), a equação nuclear de Schrödinger foi resolvida. As soluções indicam a presença de, pelo menos, três níveis vibracionais (ver Tabela 5.4). Tendo em vista que o nível vibracional $v=$ 2 encontra-se abaixo da energia de dissociação do estado fundamental do $\mathrm{BeMg}$, o que pode ser visualizado na figura ampliada da curva de energia potencial do estado $\mathrm{X}^{1} \Sigma^{+}$(Figura 5.3), podemos concluir que outros níveis devem existir. Porém, devido a problemas numéricos, não fomos capazes de determiná-los. A partir destes espaçamentos vibracionais, obtivemos um valor de $\omega_{\mathrm{e}}=44,1 \mathrm{~cm}^{-1}$ e $\omega_{\mathrm{e}} x_{\mathrm{e}}=72,6 \mathrm{~cm}^{-1}$. Os resultados obtidos a partir da análise vibracional do estado fundamental $\left(\mathrm{X}^{1} \Sigma^{+}\right)$do BeMg, e também para todos os outros estados que foram descritos neste trabalho, estão compilados nas Tabelas 5.5 e 5.4. É importante ressaltar que esta é a primeira vez que é feita uma análise vibracional para este estado.

Em torno da distância internuclear de equilíbrio, a configuração eletrônica dominante do estado fundamental [c]5 $5 \sigma^{2} 6 \sigma^{2}(0.89)$ ([c] representa os orbitais duplamente ocupados; ver Tabela 5.3); nas Figuras 5.4, 5.5 e 5.6, apresentamos a secção transversal no plano xz dos orbitais moleculares $5 \sigma, 6 \sigma$ e $7 \sigma$, respectivamente, para a distância internuclear de equilíbrio. 


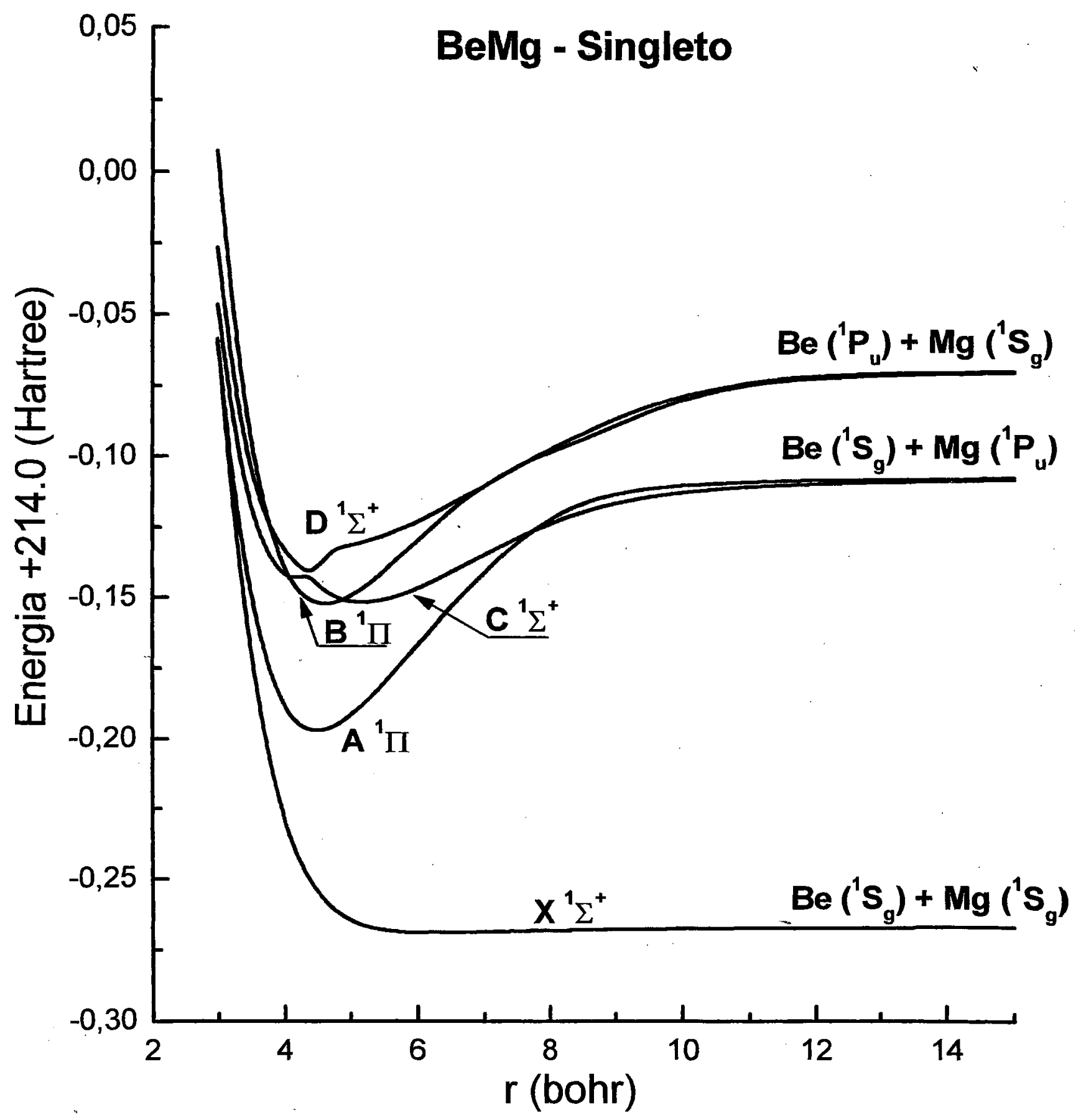

Figura 5.1: Curvas de energia total para os estados singletos da molécula BeMg. 


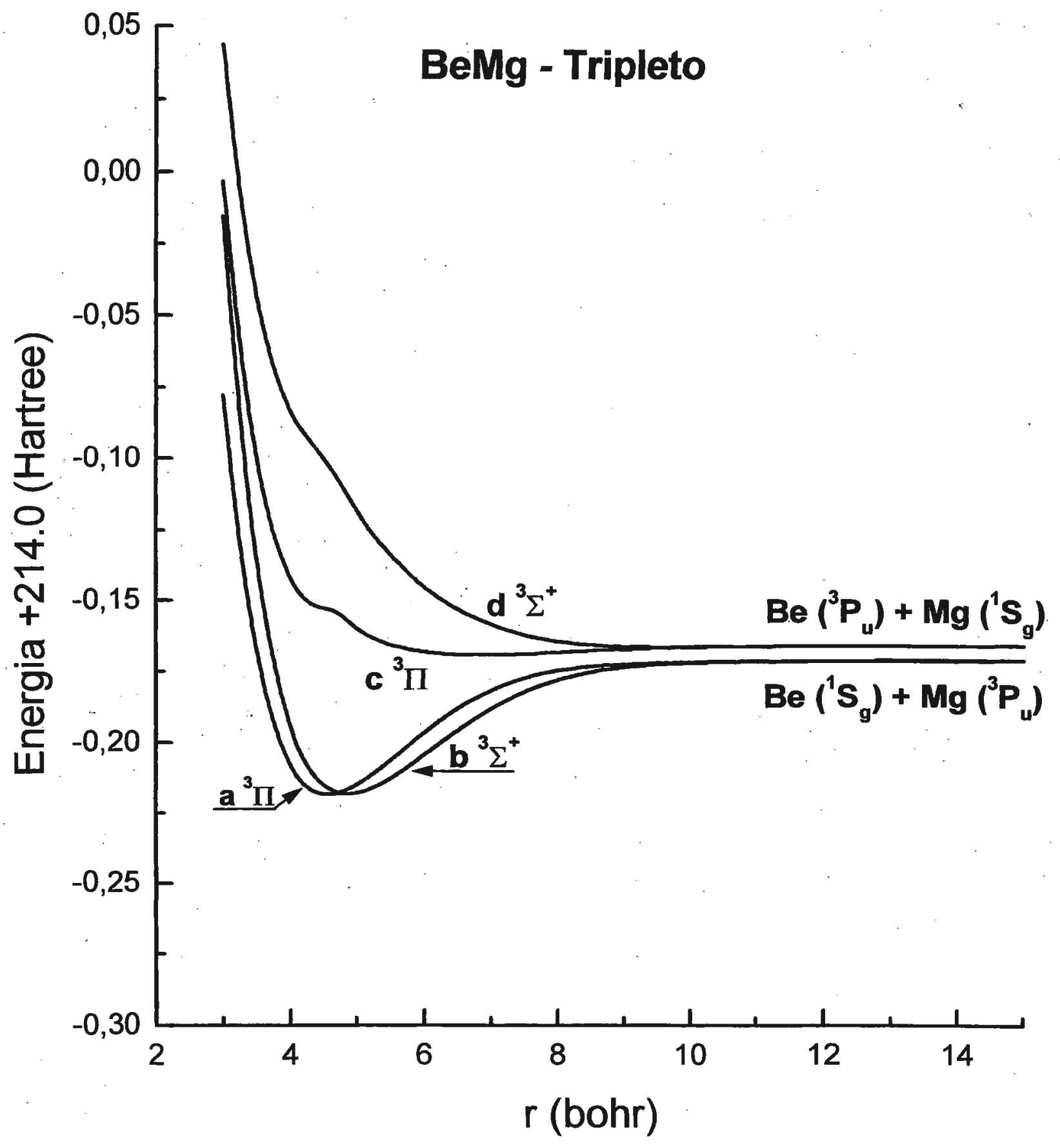

Figura 5.2: Curvas de energia total para os estados tripletos da molécula BeMg. 


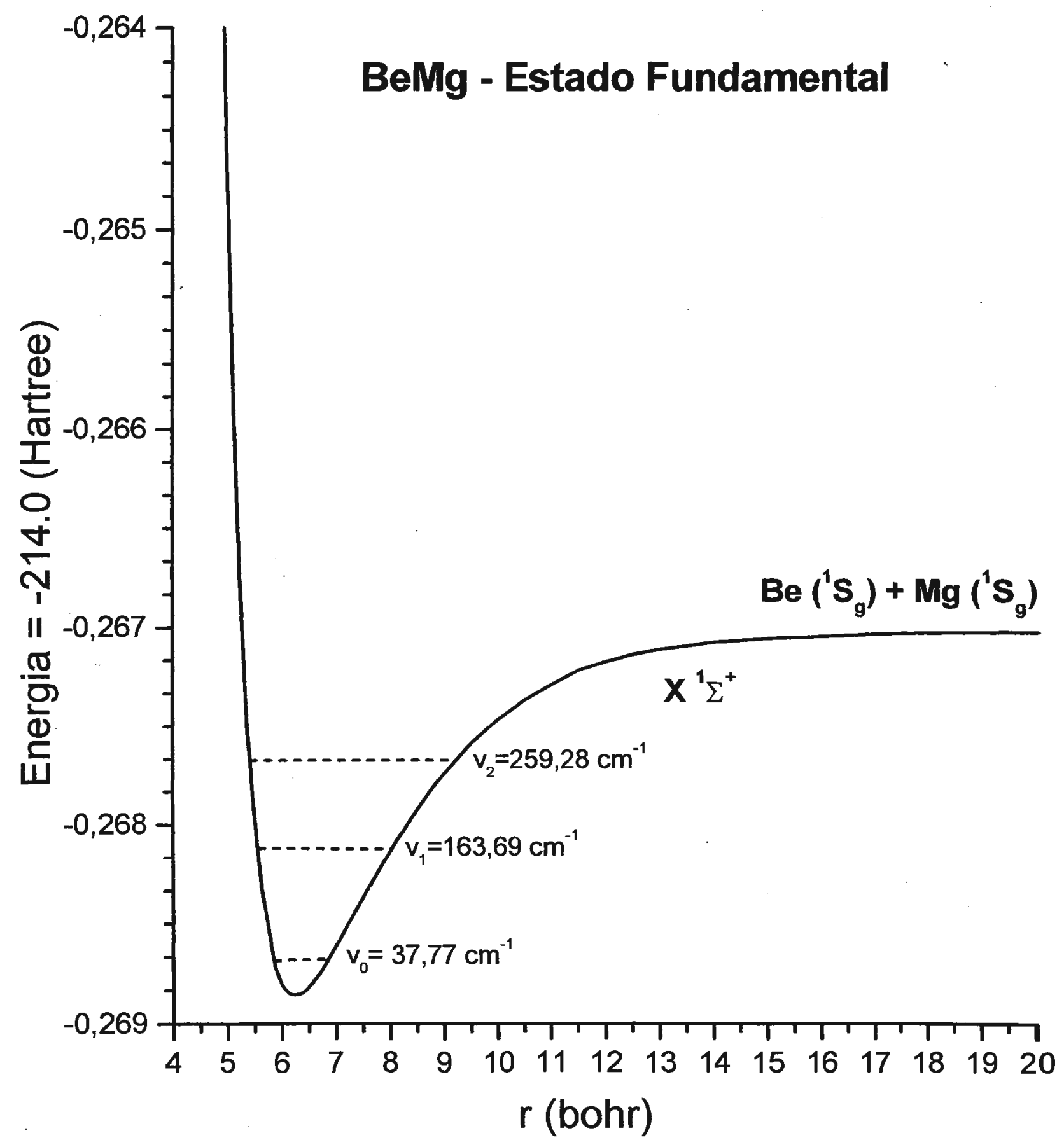

Figura 5.3: Curva de energia total para o estado fundamental da molécula BeMg. 
Tabela 5.4: Espaçamentos vibracionais $\Delta_{v+1 / 2}\left(\mathrm{~cm}^{-1}\right)$ para os estados eletrônicos de menor energia da molécula BeMg.

\begin{tabular}{crrrcccc}
\hline \hline $\mathrm{v}$ & $\mathrm{X}^{1} \Sigma^{+}$ & $\mathrm{a}^{3} \Pi$ & $\mathrm{b}^{3} \Sigma^{+}$ & $\mathrm{A}^{1} \Pi$ & $\mathrm{B}^{1} \Pi$ & $\mathrm{C}^{1} \Sigma^{+}$ & $\mathrm{D}^{1} \Sigma^{+}$ \\
\hline 0 & 125,9 & 425,9 & 388,6 & 463,6 & 430,3 & 285,7 & 768,6 \\
1 & 95,6 & 419,3 & 383,4 & 459,0 & 425,1 & 282,8 & 565,1 \\
2 & & 413,1 & 378,2 & 454,3 & 419,9 & 279,7 & 417,6 \\
3 & & 406,6 & 373,0 & 449,7 & 414,7 & 276,2 & 281,0 \\
4 & & 400,2 & 367,6 & 445,1 & 409,3 & 271,8 & 284,9 \\
5 & & 393,6 & 362,2 & 440,5 & 404,3 & 264,9 & 280,4 \\
6 & & 387,0 & 356,6 & 435,9 & 399,0 & 240,5 & 269,7 \\
7 & & 380,3 & 350,9 & 431,4 & 393,7 & 149,7 & 273,5 \\
8 & & 373,5 & 345,0 & 427,0 & 388,4 & 182,0 & 270,2 \\
9 & & 366,6 & 339,2 & 422,5 & 383,1 & 228,2 & 266,7 \\
\hline $\mathrm{E}_{0}$ & 37,8 & 217,8 & 196,3 & 234,1 & 217,6 & 144,1 & 475,6 \\
\hline \hline
\end{tabular}

Tabela 5.5: Constantes espectroscópicas $\left(\mathrm{em} \mathrm{cm}^{-1}\right)$ para os diversos estados eletrônicos da molécula BeMg.

\begin{tabular}{lccccrrr}
\hline \hline Estado & $\mathrm{T}_{\mathrm{e}}\left(\mathrm{cm}^{-1}\right)$ & $\mathrm{T}_{\mathbf{0}}\left(\mathrm{cm}^{-1}\right)$ & $\mathrm{R}_{\mathrm{e}}\left(\mathrm{a}_{0}\right)$ & $\mathrm{D}_{\mathrm{e}}(\mathrm{eV})$ & $\omega_{\mathrm{e}}$ & $\mu(\mathrm{D})$ & $\omega_{\mathrm{e}} x_{\mathrm{e}}$ \\
\hline $\mathrm{X}^{1} \Sigma^{+}$ & 0.00 & 0.000 & 6,234 & 0,05 & 44,1 & 0,219 & 72,6 \\
$\mathrm{a}^{3} \Pi$ & 11.074 & 11.252 & 4,555 & 1,28 & 432,3 & 1,640 & $-3,1$ \\
$\mathrm{~b}^{3} \Sigma^{+}$ & 11.103 & 11.261 & 4,874 & 1,28 & 393,6 & 1,104 & $-2,5$ \\
$\mathrm{~A}^{1} \Pi$ & 15.707 & 15.903 & 4,492 & 2,43 & 468,7 & 1,475 & $-2,4$ \\
$\mathrm{~B}^{1} \Pi$ & 25.522 & 25.701 & 4,614 & 2,23 & 435,7 & 1,446 & 12,6 \\
$\mathrm{C}^{1} \Sigma^{+}$ & 25.652 & 25.758 & 5,170 & 1,18 & 287,8 & 0,453 & 1,1 \\
$\mathrm{D}^{1} \Sigma^{+}$ & 27.974 & 28.411 & 4,370 & 1,93 & 930,8 & $-1,354$ & $-101,7$ \\
\hline \hline
\end{tabular}




\section{Orbital $5 \sigma$}

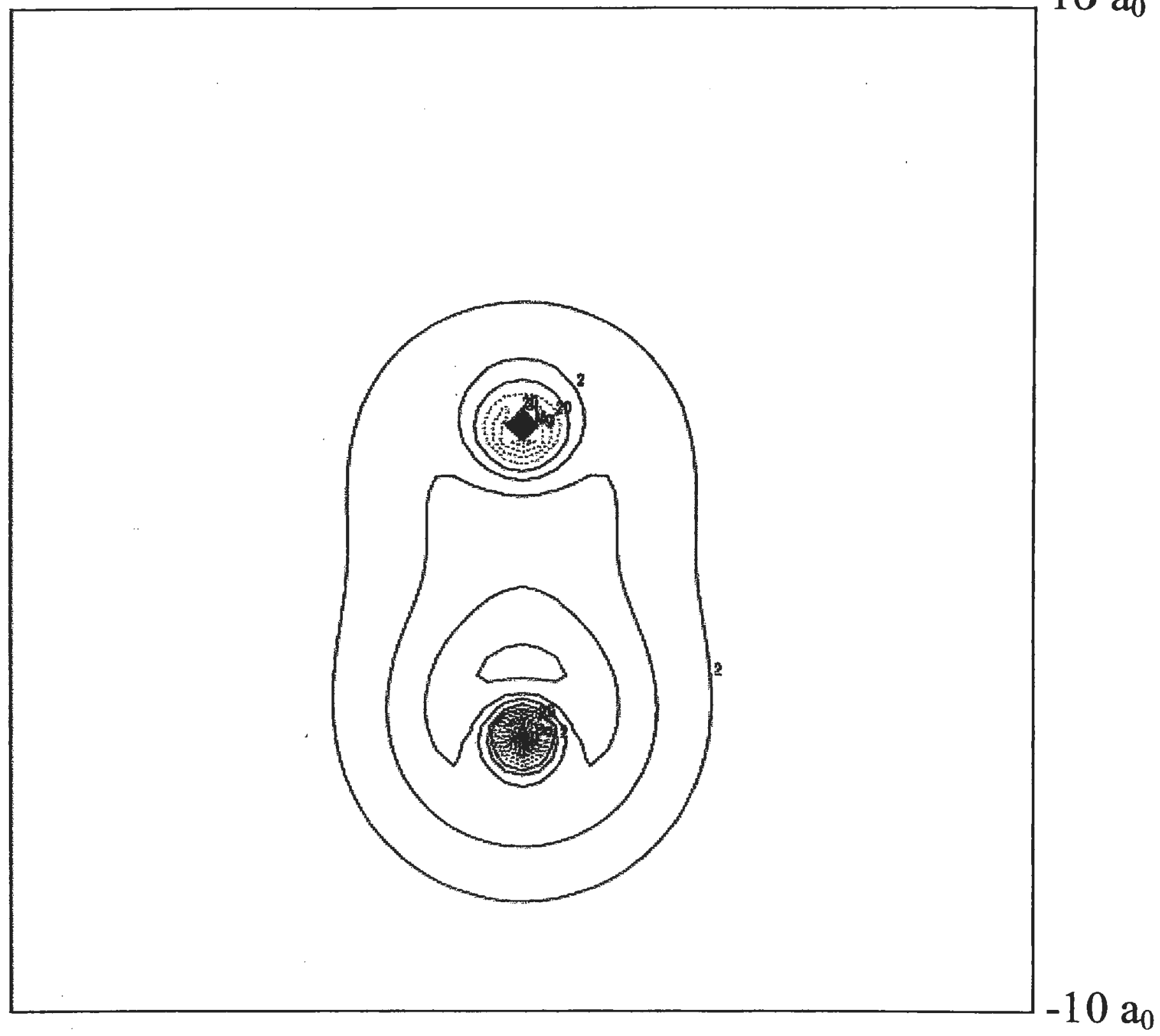

Figura 5.4: Seção transversal no plano xz dos orbital molecular $5 \sigma$, em torno da distância internuclear de equilíbrio. 


\section{Orbital $6 \sigma$}

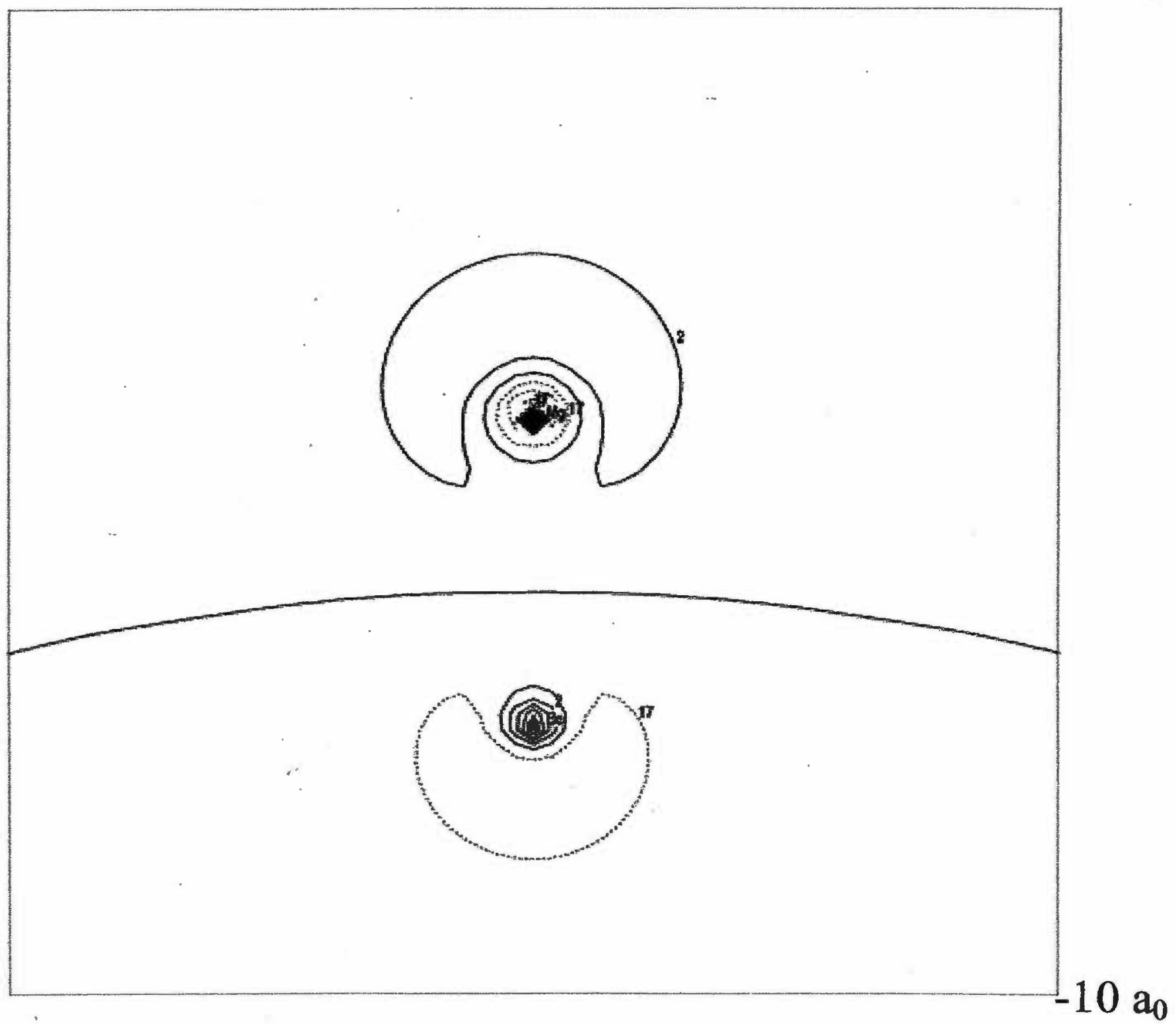

Figura 5.5: Secção transversal no plano xz do orbital molecular $6 \sigma$, em torno da posição internuclear de equilíbrio. 


\section{Orbital $7 \sigma$}

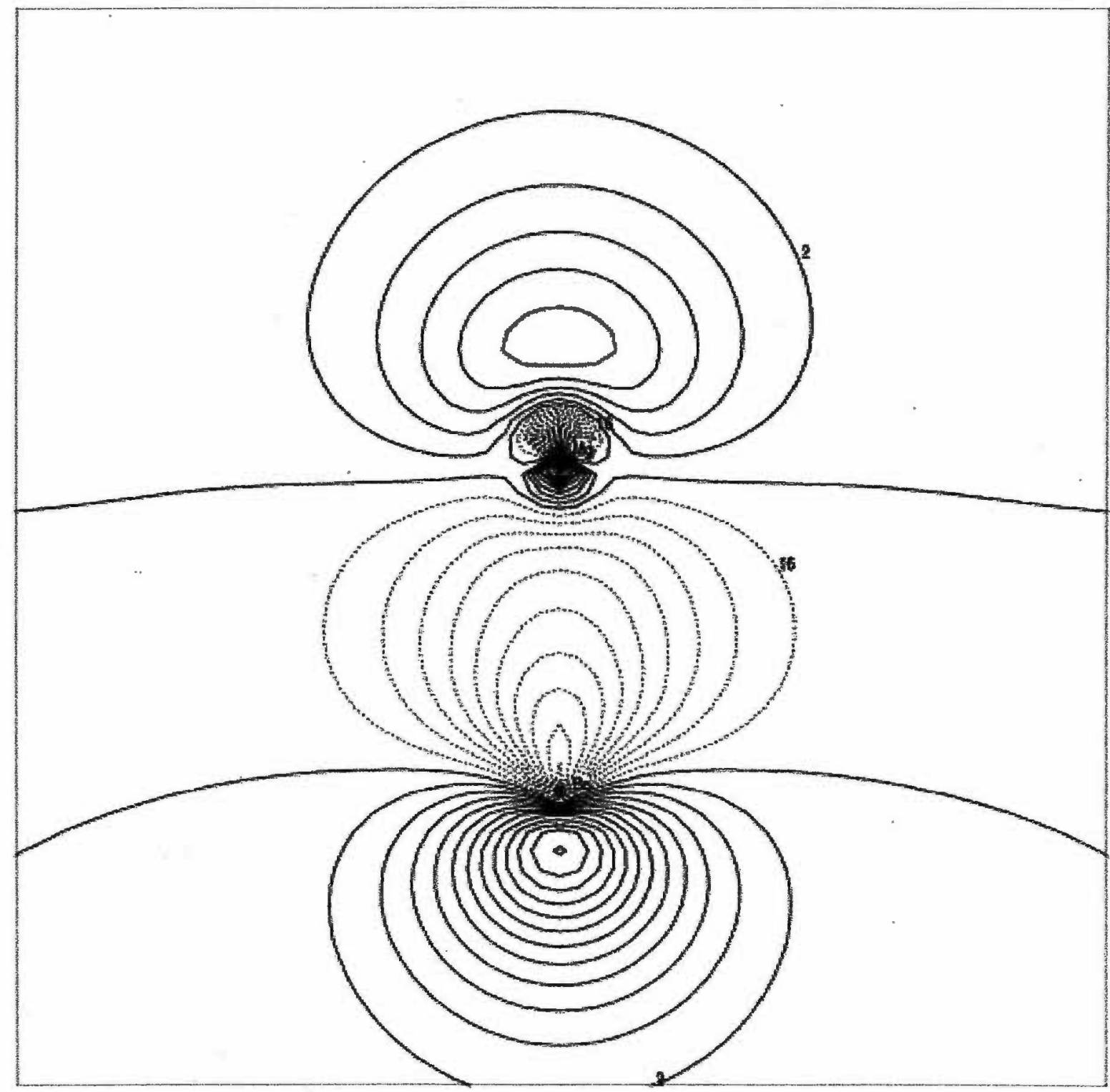

$10 \mathrm{a}_{0}$

Figura 5.6: Secção transversal no plano xz do orbital molecular $7 \sigma$, em torno da posição internuclear de equilíbrio. 
Como podemos observar, o orbital $5 \sigma$ é um orbital ligante, formando, principalmente, pela combinação dos orbitais atômicos $2 s$ do $\mathrm{Be}$ e $3 s$ do $\mathrm{Mg}$, com a densidade de carga ligeiramente distorcida para o átomo de Be. O orbital $6 \sigma$ corresponde à combinação antiligante destes mesmo orbitais atômicos. Como os dois orbitais estão duplamente ocupados, podemos supor que a ligação química entre os átomos de $\mathrm{Be}$ e $\mathrm{Mg}$ é muito fraca.

Nossos resultados para o estado fundamental da espécie BeMg podem ser comparados com outros existentes na literatura para espécies semelhantes [5,54]. Como era prevíamos, a distância internuclear de equilíbrio do $\mathrm{BeMg}\left(\mathrm{R}_{\mathrm{e}}=6,234 \mathrm{a}_{0}\right)$ é intermediária à das moléculas $\mathrm{Be}_{2}\left(4,66 \mathrm{a}_{0}\right)$ e $\mathrm{Mg}_{2}\left(7,40 \mathrm{a}_{0}\right)$. O valor de energia de dissociação do $\mathrm{BeMg}(0,05 \mathrm{eV})$ é mais próximo daquele do $\mathrm{Mg}_{2}(0,048 \mathrm{eV})$ do que do $\mathrm{Be}_{2}(0,117 \mathrm{eV})$. O orbital ligante do BeMg é levemente polarizado em direção ao Be, conforme mostrado na Figura 5.4, sendo formado basicamente pela combinação linear dos orbitais atômicos $2 \mathrm{~s}$ do berilio com o 3s do magnésio. No entanto, o orbital antiligante é polarizado levemente em direção ao $\mathrm{Mg}$, sendo formado pelos mesmo orbitais atômicos mencionados anteriormente, porém com uma maior contribuição do orbital atômico $3 p$ do magnésio, indicando que poderia ocorrer uma pequena hibridização dos orbitais $3 \mathrm{~s}$ e $3 \mathrm{p}$ do $\mathrm{Mg}$.

\subsubsection{Os Estados $\mathbf{a}^{3} \Pi \mathbf{e} \mathbf{b}^{3} \Sigma^{+}$}

O estado excitado energeticamente mais baixo da espécie BeMg é o $\mathrm{a}^{3} \Pi$, obtido a partir da excitação simples $6 \sigma \rightarrow 2 \pi$, situando-se $\left(\mathrm{T}_{e}\right) 11074 \mathrm{~cm}^{-1}$ acima do estado fundamental. De acordo com as regras de Wigner-Witmer, o estado $a^{3} \Pi$ correlaciona-se com o primeiro canal de dissociação $\left(\mathrm{Be}\left({ }^{1} \mathrm{~S}_{g}\right)+\mathrm{Mg}\left({ }^{3} \mathrm{P}_{u}\right)\right)$, localizado experimentalmente $2,71 \mathrm{eV}$ acima do canal de dissociação do estado fundamental $\left(\left(\mathrm{Be}\left({ }^{1} \mathrm{~S}_{g}\right)+\mathrm{Mg}\left({ }^{1} \mathrm{~S}_{g}\right)\right)\right.$ (Tabela 5.1). Neste trabalho, o valor calculado para esta grandeza é de $2,60 \mathrm{eV}$, em boa concordância com o valor experiemental.

Em torno da distância internuclear de equilíbrio $\left(R_{e}=4,555 a_{0}\right)$, a função de onda do estado $\mathrm{a}^{3} \Pi$ é dominada pela configuração eletrônica $[\mathrm{c}] 5 \sigma^{2} 6 \sigma^{1} 2 \pi^{1}(0,92)$. A análise vibracional indica a existência de vários níveis vibracionais (Tabela 5.4), a partir dos quais obtemos o valor de $\omega_{e}=432$ $\mathrm{cm}^{-1} \mathrm{e} \omega_{e} y_{e}=-3,1 \mathrm{~cm}^{-1}$ (Tabela 5.5). A secção transversal do orbital $2 \pi$, em torno da posição internuclear de equilíbrio, é apresenta na Figura 5.7.

Analisando a Figura 5.7, podemos concluir que o orbital molecular $2 \pi$ é um orbital ligante, polarizado em direção ao átomo de magnésio. O caráter ligante pode ser confirmado pela variação da distância internuclear de equilíbrio associada à excitação simples $6 \sigma \rightarrow 2 \pi$, que dá origem ao estado $a^{3} \Pi$, resultando em uma redução de aproximadamente $2,0 a_{0}$ em relação ao valor de $R_{e}$ do estado fundamental $\left(6,234 \mathrm{a}_{0}\right)$, que possui dois elétrons no orbital antiligante $6 \sigma$. A energia de dissociação do estado $\mathrm{a}^{3} \Pi$ é de $1,28 \mathrm{eV}$, contra $0,05 \mathrm{eV}$ para o estado fundamental. Esse fato também indica que a ligação química no estado $a^{3} \Pi$ é bem mais forte do que no estado fundamental. $O$ momento de dipolo do estado $\mathrm{a}^{3} \Pi$ é de 1,640 D.

Dissociando neste mesmo canal de dissociação, temos o segundo estado excitado da espécie $\mathrm{BeMg}$, que é o $\mathrm{b}^{3} \Sigma^{+}$; assim como no caso do estado $\mathrm{a}^{3} \Pi$, obtivemos uma boa concordância entre os valores teóricos e experimentais $(2,71 \mathrm{eV}$ e $2,60 \mathrm{eV}$, respectivamente) para a posição do limite de dissociação (Tabela 5.1). Localizado $11103 \mathrm{~cm}^{-1}$ acima do estado fundamental, ele é obtido a partir do estado fundamental por uma excitação simples $6 \sigma \rightarrow 7 \sigma$, sendo a função de onda predominantemente 


\section{Orbital $2 \pi$}

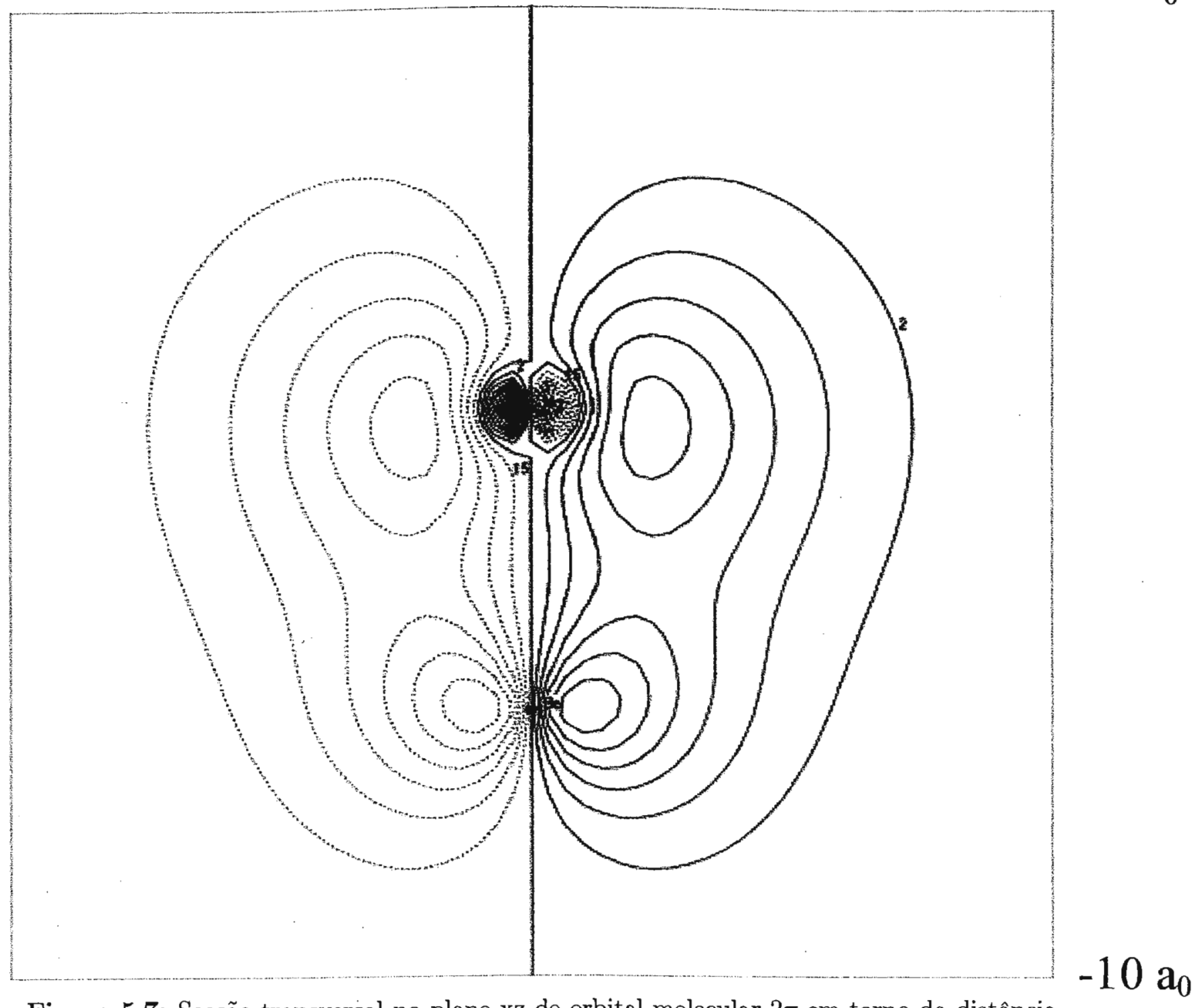

Figura 5.7: Seç̧ão transversal no plano xz do orbital molecular $2 \pi$ em torno da distância internuclear de equilíbrio. 
representada pela configuração eletrônica $[c] 5 \sigma^{2} 6 \sigma^{1} 7 \sigma^{1}(0,95)$. A distância internuclear de equílibrio é de 4,874 a e a energia de dissociação calculada igual a $1,28 \mathrm{eV}$ e o momento de dipolo igual a 1,104 D. Como a distância internuclear de equílibrio do estado $a^{3} \Pi$ é muito menor do que a do estado fundamental, e energia de dissociação muito superior ao do estado $\mathrm{X}^{1} \Sigma^{+}$, podemos dizer que a ligação química existente no estado $\mathrm{a}^{3} \Pi$ é mais forte do que a do estado fundamental.

Resolvendo-se a equação nuclear de Schrödinger, obtivemos os espaçamentos vibracionais mostrados na Tabela 5.4, a partir dos quais foram obtidas as constantes espectroscópicas apresentadas na Tabela 5.5.

\subsubsection{Os Estados $\mathrm{A}^{1} \Pi$ e $\mathrm{C}^{1} \Sigma^{+}$}

Os primeiros estados excitados de simetria de spin singleto são os $\mathrm{A}^{1} \Pi$ e $\mathrm{C}^{1} \Sigma^{+}$, que estão correlacionados com o quarto canal de dissociação $\mathrm{Be}\left({ }^{1} \mathrm{~S}_{g}\right)+\mathrm{Mg}\left({ }^{1} \mathrm{P}_{u}\right)$ (Tabela 5.1). Experimentalmente, este canal está localizado $4,34 \mathrm{eV}$ acima do primeiro canal de dissociação ( $\operatorname{Be}\left({ }^{1} \mathrm{~S}_{g}\right)+\mathrm{Mg}\left({ }^{1} \mathrm{~S}_{g}\right)$ ), sendo que o valor obtido em nosso trabalho é de $4,31 \mathrm{eV}$ e 4,32 eV, para os estados $\mathrm{A}^{1} \Pi$ e $\mathrm{C}^{1} \Sigma^{+}$, respectivamente, em excelente concordância com os valores experimentais.

O estado $\mathrm{A}^{1} \Pi$, cujo momento de dipolo calculado é de $1,474 \mathrm{D}$, é descrito pela mesma distribuição eletrônica espacial do estado $a^{3} \Pi$, diferindo obviamente no acoplamento de spin. Ele é obtido a partir de uma excitação simples do estado fundamental $(6 \sigma \rightarrow 2 \pi)$, sendo dominado por uma única configuração eletrônica em torno da região internuclear de equilíbrio: [c]5 $5 \sigma^{2} 6 \sigma^{1} 2 \pi^{1}(0,89)$. Energeticamente, ele está situado $15707 \mathrm{~cm}^{-1}\left(\mathrm{~T}_{e}\right)$ acima do estado fundamental, com uma energia de dissociação igual a 2,43 eV. A análise vibracional indica a existência de vários níveis vibracionais (Tabela 5.4), a partir dos quais obtivemos para $\omega_{\mathrm{e}}$ o valor de $468,7 \mathrm{~cm}^{-1}$ (Tabela 5.5). Devido à natureza dos orbitais moleculares envolvidos, podemos concluir que a ligação química existente no estado $\mathrm{A}^{1} \Pi$ é semelhante a do estado $\mathrm{a}^{3} \Pi$.

O estado $\mathrm{C}^{1} \Sigma^{+}$situa-se a $25652 \mathrm{~cm}^{-1}$ acima do estado fundamental, com uma distância internuclear de equilíbrio igual a 5,170 a a, energia de dissociação de $1,18 \mathrm{eV}$ e momento de dipolo igual a 0,453 D. Por sua vez, a função de onda que descreve o estado $C^{1} \Sigma^{+}$é dominada por, pelo menos, duas configurações eletrônicas, [c] $5 \sigma^{2} 6 \sigma^{2}(0,58)$ e $[\mathrm{c}] 5 \sigma^{2} 7 \sigma^{2}(0,29)$, na parte repulsiva da curva de energia potencial; na parte atrativa, as configurações $[\mathrm{c}] 5 \sigma^{2} 6 \sigma^{1} 7 \sigma^{1}(0,70)$ e $[\mathrm{c}] 5 \sigma^{2} 7 \sigma^{2}(0,38)$ passam a dominar a função de onda. O valor de $\omega_{\mathrm{e}}$ para este estado é de $287,8 \mathrm{~cm}^{-1}\left(\omega_{\mathrm{e}} x_{\mathrm{e}}=1,1 \mathrm{~cm}^{-1}\right)$ (Tabela 5.5) e os primeiros espaçamentos vibracionais estão apresentados na Tabela 5.4.

\subsubsection{Os Estados $\mathrm{B}^{1} \Pi$ e $\mathrm{D}^{1} \Sigma^{+}$}

Os estados $\mathrm{B}^{1} \Pi$ e $\mathrm{D}^{1} \Sigma^{+}$estão correlacionados com o quinto canal de dissociação $\left(\mathrm{Be}\left({ }^{1} \mathrm{P}_{u}\right)+\right.$ $\mathrm{Mg}\left({ }^{1} \mathrm{~S}_{g}\right)$ ) que, experimentalmente, está localizado $5,27 \mathrm{eV}$ acima do canal de dissociação do estado fundamental. No limite de dissociação, nos cálculos posicionam os estados $\mathrm{B}^{1} \Pi$ e $\mathrm{D}^{1} \Sigma^{+}$em 5,34 acima do canal de dissociação do estado fundamental. Novamente, a concordância com o valor experimental é boa.

$O$ estado $B^{1} \Pi$ é um estado gerado a partir de um excitação dupla do estado fundamental $\left(6 \sigma^{2} \rightarrow\right.$ $\left.7 \sigma^{1} 2 \pi^{1}\right)$, sendo que duas configurações dominam a função de onda eletrônica em torno da distância

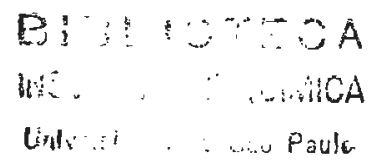


internuclear de equilíbrio: [c] $5 \sigma^{2} 7 \sigma^{1} 2 \pi^{1}(0,75)$ e $[\mathrm{c}] 5 \sigma^{2} 6 \sigma^{1} 3 \pi^{1}(0,43)$. Localizando-se a $\left(\mathrm{T}_{e}\right) 25522$ $\mathrm{cm}^{-1}$ acima do estado fundamental, sua distância internuclear de equílibrio é de 4,614 a o e energia de dissociação $2,23 \mathrm{eV}$, o que nos indica uma ligação química mais forte que no estado fundamental e semelhante a dos outros estados excitados. A partir dos espaços vibracionais mostrados na Tabela 5.4 , obtivemos um valor de $\omega_{\mathrm{e}}=435,7 \mathrm{~cm}^{-1}$, com um correspondente $\omega_{\mathrm{e}} x_{\mathrm{e}}=12,6 \mathrm{~cm}^{-1}$. Para o estado $\mathrm{B}^{1} \mathrm{I}$; o momento de dipolo foi calculado com sendo igual a 1,446 D.

$O$ estado excitado mais alto energeticamente é o $D^{1} \Sigma^{+}$, localizado a $27974 \mathrm{~cm}^{-1}$ acima do estado fundamental, tendo uma distância internuclear de equilíbrio igual a 4,370 $\mathrm{a}_{0}$ e momento de dipolo igual a $-1,353 \mathrm{D}$. Assim como o estado $\mathrm{C}^{1} \Sigma^{+}$, o estado $\mathrm{D}^{1} \Sigma^{+}$também é descrito por duas configurações eletrônicas na região repulsiva da curva de energia potencial próxima à distância internuclear de equilíbrio, [c] $5 \sigma^{2} 2 \pi^{2}(0,57)$ e $[\mathrm{c}] 5 \sigma^{2} 6 \sigma^{2}(0,39)$, sendo que a predominância muda para as configurações [c] $5 \sigma^{2} 6 \sigma^{1} 7 \sigma^{1}(0,45)$, [c]5 $5 \sigma^{2} 7 \sigma^{2}(0,43)$ e $[c] 5 \sigma^{2} 2 \pi^{2}(0,35)$ na região atrativa nas imediações da posição de equilíbrio. Da análise vibracional (Tabela 5.4), obtemos que $\omega_{\mathrm{e}}=930,8 \mathrm{~cm}^{-1} \mathrm{e}$ $\omega_{\mathrm{e}} x_{\mathrm{e}}=-101,7 \mathrm{~cm}^{-1}$, indicando que este estado é fortemente perturbado.

\subsubsection{Análise dos Orbitais Moleculares}

Conforme frisamos anteriormente, os orbitais moleculares $5 \sigma, 6 \sigma, 7 \sigma$ e $2 \pi$ (Figs. 5.4, 5.5, 5.6 e 5.7, respectivamente) são os mais importantes para a análise dos estados eletrônicos descritos neste trabalho. Em torno da posição internuclear de equilíbrio, o orbital $5 \sigma$ é ligante, formado pela combinação dos orbitais atốmicos $2 s$ do Be e $3 s$ do $\mathrm{Mg}$, com a densidade de carga ligeiramente distorcida para o átomo de Be, enquanto o $6 \sigma$ corresponde à combinação antiligante. Portanto, durante o processo de dissociação da espécie $\mathrm{BeMg}$, estes orbitais moleculares deverão reproduzir os orbitais atômicos $2 s$ do Be e $3 s$ do $\mathrm{Mg}$. A Figura 5.8 ilustra claramente este fato.Conforme podemos observar, a medida que a distância internuclear entre os átomos de $\mathrm{Be}$ e $\mathrm{Mg}$ vai aumentando, os orbitais moleculares $5 \sigma$ e $6 \sigma$ vão se transformando no orbitais atômicos $2 s$ do $\mathrm{Be}$ e $3 s$ do $\mathrm{Mg}$, conforme era previsto. Análise semelhante pode ser feita para os outros dois orbitais moleculares.

\subsection{Conclusões}

Neste trabalho, a espécie diatômica BeMg foi estudada empregando-se o método ab inito Interação de Configurações Multiconfiguracional (MRCI), empregando como função de onda do tipo CASSCF (complete active space self-consistent field) como função de ordem zero, e extensos conjuntos de bases atômicas do tipo cc-pVQZ. No espaço ativo foram incluídos todos os elétrons e orbitais de valência dos átomos de $\mathrm{Be} \mathrm{e} \mathrm{Mg}$, além de um conjunto de orbitais de correlação, contendo funções do tipo $s$ e $p$. Ao contrário de estudos realizados anteriormente, nos quais apenas o estado fundamental foi descrito, empregando-se bases atômicas menos flexíveis e métodos químico-quânticos mais aproximados, neste trabalho descrevemos cuidadosamente o estado fundamental e 8 estados eletrônicos excitados (singletos e tripletos).

Dentre todos os estados eletrônicos estudados, $\mathrm{c}^{3} \mathrm{\Pi I}$ e d ${ }^{3} \Sigma^{+}$não são ligados. Os outros estados estão dispostos em ordem crescente de energia de excitação, da seguinte forma: $X^{1} \Sigma^{+}\left(R_{e}=6,234\right.$ $\left.a_{0}\right), a^{3} \Pi\left(R_{e}=4,555 a_{0}, T_{e}=11.074 \mathrm{~cm}^{-1}\right), b^{3} \Sigma^{+}\left(R_{e}=4,874 a_{0}, T_{e}=11.103 \mathrm{~cm}^{-1}\right), A^{1} \Pi$ 

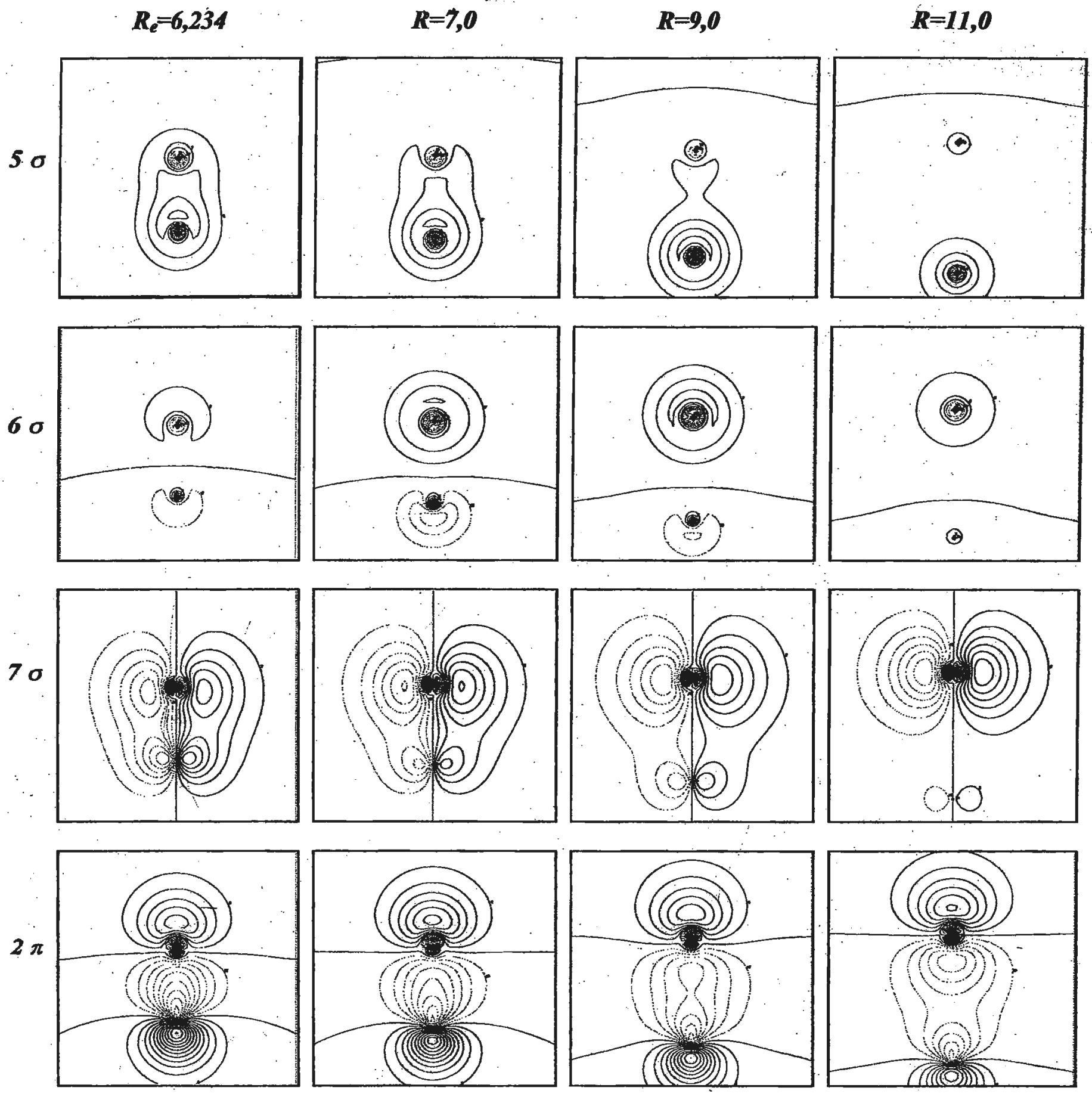

Figura 5.8: Secção transversal no plano xz dos orbitais moleculares $5 \sigma, 6 \sigma, 7 \sigma$ e $2 \pi$ para diferentes distâncias internucleares. 
$\left(\mathrm{R}_{\mathrm{e}}=4,492 \mathrm{a}_{0}, \mathrm{~T}_{\mathrm{e}}=15.707 \mathrm{~cm}^{-1}\right), \mathrm{B}^{1} \Pi\left(\mathrm{R}_{\mathrm{e}}=4,614 \mathrm{a}_{0}, \mathrm{~T}_{\mathrm{e}}=25.522 \mathrm{~cm}^{-1}\right), \mathrm{C}^{1} \Sigma^{+}\left(\mathrm{R}_{\mathrm{e}}=5,170 \mathrm{a}_{0}\right.$, $\left.\mathrm{T}_{\mathrm{e}}=25.652 \mathrm{~cm}^{-1}\right)$ e $\mathrm{D}^{1} \Sigma^{+}\left(\mathrm{R}_{\mathrm{e}}=4,370 \mathrm{a}_{0}, \mathrm{~T}_{\mathrm{e}}=27.974 \mathrm{~cm}^{-1}\right)$.

$\mathrm{O}$ estado fundamental do BeMg é de simetria ${ }^{1} \Sigma^{+}$, em concordância com trabalhos anteriores. Sua distância internuclear de equilíbrio igual é de $6,234 \mathrm{a}_{0}(3,30 \AA)$, inferior aquela obtida anteriormente por Chiles e Dykstra $(4,5 \AA)$ e Boldyrev et. al $(5,1 \AA)$. A energia de dissociação foi calculada como sendo igual a $0,05 \mathrm{eV}(1,2 \mathrm{kcal} / \mathrm{mol})$. Como a energia de dissociação é pequena, empregamos o método conhecido como counterpoise correction para estimar o erro de sobreposição de base, obtendo um valor corrigido numericamente igual àquele sem a correção. Isso indica que não há erro de sobreposição de base. Portanto, podemos concluir que na distância internuclear de $3,30 \AA$, os átomos unidos são mais estáveis que separados. A partir da solução numérica da equação nuclear de Schrödinger para o estado fundamental, identificamos a existência de três níveis vibracionais para este estado, fato até então totalmente desconhecido teórica e experimentalmente. A partir destes espaçamentos vibracionais, obtivemos um valor de $\omega_{\mathrm{e}}=44,1 \mathrm{~cm}^{-1}$ e $\omega_{\mathrm{e}} x_{\mathrm{e}}=72,6 \mathrm{~cm}^{-1}$.

Em torno da distância internuclear de equilíbrio, a configuração eletrônica que domina a descrição do estado fundamental é $5 \sigma^{2} 6 \sigma^{2}$, que participa com um coeficiente de 0,89 na espansão CI. O orbital $5 \sigma$ é um orbital ligante, formando, principalmente, pela combinação dos orbitais atômicos $2 s$ do Be e $3 s$ do $\mathrm{Mg}$, com a densidade de carga ligeiramente distorcida para o átomo de $\mathrm{Be}$; o $6 \sigma$ corresponde à combinação antiligante destes mesmo orbitais atômicos. $\mathrm{O}$ fato dois orbitais estarem duplamente ocupados, indica que a ligação química entre os átomos de Be e $\mathrm{Mg}$ é muito fraca, conforme podemos concluir a partir da distância internuclear de equilíbrio e da energia de dissociação para o estado fundamental. 


\section{Bibliografia}

[1] E. Clementi, G. Corongiu, D. Bahattacharya, B. Feuston, D. Frye, A. Preiskorn, A. Rizzo e W. Xue, Chem. Rev. 91, 679 (1991).

[2] F. R. Ornellas, O. Roberto-Neto, A. C. Borin e F. B. C. Machado, J. Chem. Phys. 95, 9086 (1991).

[3] A. C. Borin e F. R. Ornellas, J. Chem. Phys. 98, 8761 (1993).

[4] F. R. Ornellas e A. R. S. Valentim, J. Phys. Chem. 98, 12570 (1994).

[5] R. A. Chiles e C. E. Dykstra, Chem. Phys. Lett. 85, 447 (1982).

[6] A. I. Boldyrev, N. Gonzales e J. Simons, J. Phys. Chem. 98, 9931 (1994).

[7] H. J. Lugauer, F. Fischer, T. Litz, A. WaAg, U. Zehnder, W. Ossau, T. Gerhard, G. LANDWeHr, C. BECKer, R. KRUSE e J. Geurts, Materials Science and Engineering B43, 88 (1997).

[8] A. Szabo e N. S. Ostlund, Modern Quantum Chemistry. Introduction to Advanced Electronic Structure Theory, $1^{a}$ edição revisada, McGraw-Hill, NY, USA, 1989.

[9] J. AlmLöF, Notes on Hartree-Fock Theory, em Lecture Notes in Quantum Chemistry, European. Summer School in Quantum Chemistry, em Lecture Notes in Chemistry, 58 (B. O. Roos, editor), p. 1, Springer-Verlag, Berlin, Germany, 1992.

[10] F. Jensen, Introduction to Computational Chemistry, John Wiley \& Sons, England, 1999.

[11] C. C. J. RoothaAn, Rev. Mod. Phys. 23, 69 (1951).

[12] G. C. Hall, Proc. R. Soc. (London) A205, 541 (1951).

[13] W. J. Hehre, R. F. Stewart e J. A. Pople, J. Chem. Phys. 51, 2657 (1969).

[14] J. S. Binkley e J. A. Pople, J. Am. Chem. Soc. 102, 939 (1980).

[15] W. J. Hehre, R. Ditchfield e J. A. Pople, J. Chem. Phys. 56, 2257 (1972).

[16] R. Krishnan, J. S. Binkley, R. Seeger e J. A. Pople, J. Chem. Phys. 72, 650 (1980).

[17] M. J. Frisch, J. A. Pople e J. S. Binkley, J. Chem. Phys. 80, 3265 (1984). 
[18] M. M. Francl, W. J. Pietro, W. J. Hehre, J. S. Binkley, M. S. Gordon, D. J. DeFrees e J. A. Pople, J. Chem. Phys. 77, 3654 (1982).

[19] R. C. Raffenetti, J. Chem. Phys. 58, 4452 (1973).

[20] J. Almlöf e P. R. Taylor, J. Chem. Phys. 86, 4070 (1987).

[21] J. Almlöf e P. R. Taylor, J. Chem. Phys. 92, 551 (1990).

[22] J. Almlöf e P. R. TAYlor, Atomic Natural Orbital (ANO) Basis Sets for Quantum Chemical Calculations, em Advances in Quantum Chemistry, 52, (PER-OLOV LOWDIN, editor), p. 301, Academic Press, California, USA, 1991.

[23] C. W. Bauschlicher JR., Chem. Phys. Lett. 142, 71 (1987).

[24] S. R. Langhoff, C. W. Bauschlicher Jr.e P. R. Taylor, J. Chem. Phys. 88, 5715 (1988).

[25] S. R. Langhoff, C. W. Bauschlicher JR. e P. R. Taylor, J. Chem. Phys. 89, 7650 (1988).

[26] P.-O. Widmark, P.-A. Malmqvist e B. O. Roos, Theor. Chim. Acta 77, 291 (1990).

[27] P.-O. Widmark, B. J. Persson e B. O. Roos, Theor. Chim. Acta 79, 419 (1991).

[28] C. W. Bauschlicher JR. e P. R. Taylor, Theor. Chim. Acta 86, 13 (1993).

[29] T. H. Dunning JR., J. Chem. Phys. 90, 1007 (1989).

[30] D. E. Woon e T. H. Dunning, J. Chem. Phys. 98, 1358 (1993).

[31] A. K. Wilson, T. van Mourik e T. H. Dunning JR., J. Mol. Struct. 388, 339 (1996).

[32] R. A. Kendall, T. H. Dunning JR. e R. J. Harrison, J. Chem. Phys. 96, 6796 (1992).

[33] D. E. Woon e T. H. Dunning JR., J. Chem. Phys. 103, 4572 (1995).

[34] T. H. Dunning e P. J. HaY, Gaussian Basis Sets for Molecular Calculations, em Modern Theoretical Chemistry, 3, (H. F. SCHAEFER III, editor), p. 1, Plenum Press, New York, USA, 1977.

[35] D. Feller e E. R. Davidson, Chem. Rev. 86, 681 (1986).

[36] D. Feller e E. R. Davidson, Basis Sets for Ab Initio Molecular Orbital Calculations and Intermolecular Interactions, in Reviews in Computational Chemistry, (K. B. LIPKowITZ e D. B. BoYD, editores), p. 1, VCH, New York, USA, 1990.

[37] P. O. Lowdin, Correlation Problem in Many-Electron Quantum Mechanics. I. Review of Different Approaches and Discussion of Some Current Ideas. em Advances in Chemical Physics, 2; (I. PRIGogine, editor), p. 207, John Wiley \& Sons Ltd, Chichester, England, 1959.

[38] A. C. WAHL e G. DAs, The Multiconfiguration Self-Consistent Field Method, em Modern Theoretical Chemistry, 3 (H. F. ScHAEFER III, editor), p. 51, Plenum Press, New York, USA, 1977. 
[39] B. O. Roos, The Complete Active Space Self-Consistent Field Method and Its Applications in Electronic Structure Calculations. em Advances in Chemical Physics, 69; Ab Initio Methods in Quantum Chemistry - II (K. P. LAWLEY, editor), p. 399, John Wiley \& Sons Ltd, Chichester, England, 1987.

40] B. O. Roos, The Multiconfigurational (MC) Self-Consistent Field (SCF) Theory, em Lecture Notes in Quantum Chemistry, European Summer School in Quantum Chemistry, em Lecture Notes in Chemistry, 58 (B. O. Roos, editor), p. 177, Springer-Verlag, Berlin, Germany, 1992.

41] P. E. M. Siegbahn, The Configuration Interaction Method, em Lecture Notes in Quantum Chemistry, European Summer School in Quantum Chemistry, em Lecture Notes in Chemistry, 58 (B. O. Roos, editor), p. 255, Springer-Verlag, Berlin, Germany, 1992.

42] R. McWeenY, Methods of Molecular Quantum Mechanics, Academic Press, England, 1992.

43] H. J. Werner e P. J. Knowles, J. Chem. Phys. 89, 5883 (1988).

44] P. J. Knowles e H. J. Werner, Chem. Phys. Lett. 145, 514 (1988).

45] P. J. Knowles e H. J. Werner, Chem. Phys. Lett. 115, 259 (1985).

46] H. J. Werner e P. J. Knowles, J. Chem. Phys. 82, 5053 (1985).

47] MOLPRO é um programa para cálculos ab initio escrito por H.-J. Werner e P. J. Knowles, com contribuições de R. D. Amos, A. Berning, D. L. Cooper, M. J. O. Deegan, A. J. Dobbyn, F. Eckert, C. Hampel, G. Hetzer, T. Leininger, R. Lindh, A. W. Lloyd, W. Meyer, M. E. Mura, A. Nicklaß, P. Palmieri, K. Peterson, R. Pitzer, P. Pulay, G. Rauhut, M. Schütz, H. Stoll, A. J. Stone e T. Thorsteinsson.

48] J. M. L. Martin, Chem. Phys. Lett. 303, 399 (1999).

49] K. Andersson, M. R. A. Blomberg, M. P. Fülscher, G. Karlstöm, R. Lindh, P.-Å. Malmqvist, P. Neogrády, J. Olsen, B. O. Roos, A. J. Sadlej, M. Schütz, L. Seijo, L. Serrano-Andrés, P. E. M. Siegbahn e P.-O. Widmark. MOLCAS Version 4.0. Dept. of Theor. Chem., Chem. Center, Univ. of Lund, P.O.B. 124, S-221 00 Lund, Sweden Lund (1997).

50] G. Herzberg, Molecular Spectra and Molecular Structure. Vol. I, Spectra of Diatomic Molecules, Van Nostrand Reinhold, NY, 1950.

51] C. Moore, Atomic Energy Levels, Natl. Stand. Ref. Data Ser. Natl. Bur., Technical Report 35, U. S. GPO, Washington, DC, 1971.

52] S. F. Boys e F. Bernardi, Mol. Phys. 19, 553 (1970).

53] F. B. van Duijneveldt, J. G. C. M. van Duijneveldt de Rijdt e J. H. van Lenthe, Chem. Rev. 94, 1873 (1994).

54] C. W. Bauschlicher, S. R. Langhoff e H. Partridge, J. Chem. Phys. 96, 1240 (1992). 


\section{Parte III}

\section{Apêndice}

